\title{
New Insulins, Biosimilars, and Insulin Therapy
}

\author{
Thomas Danne ${ }^{1}$, Lutz Heinemann ${ }^{2}$, and Jan Bolinder ${ }^{3}$
}

Introduction

S THE CENTENARY OF THE DISCOVERY of insulin (1921) is on the horizon, the $A$ revolutionary impact on the lives of people with diabetes mellitus will be the focus of many reviews in the near future. Even now, incremental changes to insulin preparations and insulin therapy are happening from year to year. On a worldwide scale challenges to ensure the quality of insulins in terms of purity, noncontamination with spurious proteins, and the use of human insulin for humans remain. The dawn of automated insulin delivery (AID) systems - misleadingly often referred to as "artificial pancreas"- offers even better futures for our patients. However, differences in regulatory processes and reimbursement guide the regional availability of these advances for patients. In the United States, the Food and Drug Administration (FDA) had already given the go-ahead for the so-called "hybrid closed-loop pump" in September 2016. Since then, over 100,000 pumps with the Minimed 670G system have apparently been sold there. During the Advanced Technologies and Treatments for Diabetes (ATTD) 2018 Conference held in Vienna in February 2018, 867 participants signed the Call for Action asking the industry, regulatory agencies, and health-care policy-makers to accelerate the availability of AID systems in the European Union. Eventually the manufacturer reported that he had received a CE mark for European conformity at the end of June 2018, close to 2 years after the FDA approval. However, this does not mean that this system is now actually available on all markets, let alone that a reimbursement by the health insurance is given. In countries such as Belgium, Denmark, Finland, Ireland, Italy, Netherlands, Norway, Spain, Sweden, Switzerland, and the United Kingdom, this hybrid AID system is expected to be available by the end of 2018. On the other hand, it cannot be foreseen when this will be the case, for example, in Germany. Apparently, the system is considered a new diagnostic and therapeutic method, for which a lengthy regulatory approval process must be completed in order to ensure reimbursement by the statutory health-care system for the cost of the system. Such approval processes could easily take longer than the development cycle for the next meaningful improvement in commercially available AID system.

Experimental progress has also been made regarding the smart insulin concept. Various approaches have been described in the past year, most notably by using specially designed, insulin-filled vesicles to automatically and efficiently control blood glucose levels in diabetic mice lacking beta cells. With a single injection of these so-called "artificial beta cells," a rise in blood glucose levels leads to high glucose uptake and oxidation, generating a low $\mathrm{pH}$ within these cells. This induces structural changes in the synthetic peptides tethered to the insulin loaded inner vesicles, ultimately leading to a release of insulin outside the cell. Declining $\mathrm{pH}$ as a consequence of glucose normalization would halt insulin release. These artificial cells are expected to trigger a limited immune response without the need for immunomodulation. As we have stated in previous yearbooks, it will take time until smart insulin becomes a clinical reality, but the data are encouraging, and this area will most certainly remain important for future yearbooks.

At the core of all therapeutic advances seen in the last few years, insulin remains the pillar of treatment. At the same time, during the last year, more and more data for

\footnotetext{
${ }^{1}$ Diabetes Center for Children and Adolescents, Children's Hospital AUF DER BULT, Hannover, Germany

${ }^{2}$ Science \& Co. GmbH, Neuss, Germany

${ }^{3}$ Department of Medicine, Karolinska University Hospital Huddinge, Karolinska Institutet, Stockholm, Sweden
} 
adjunct therapy with sodium-glucose cotransporter 2 inhibitors in patients with type 1 diabetes have become available and regulatory submissions are on the way, with decisions expected early next year. These drugs have the potential of addressing common side effects of insulin therapy such as glycemic variability, weight gain, or hypoglycemia. This must be balanced against notable untoward effects such as increased risk for genital mycotic infections or diabetic ketoacidosis.

Mealtime insulin should ideally be absorbed very quickly after injection to counteract the rise in blood glucose with food consumption. Regarding the next-generation mealtime insulin Fiasp (NovoNordisk), it is now available in several markets and more data from the clinical Onset trial program with FiAsp were published or presented during the last year. As we outlined in the previous yearbook, these insulins where thought to be of particular value in insulin pump therapy. However, when the results of the Onset 5 FiAsp pump trial were presented during the 2017 ATTD meeting, the results were controversial. While we are still waiting for the eventual publication of this study, Eli Lilly initiated the PRONTO-Pump phase 3 trial to evaluate the compatibility and safety of their ultrarapid formulation LY 900014 or trepostinil lispro compared with insulin lispro in February 2018.

Although clinical data of Adocia's ultra-rapid insulin-known as BioChaperone Lispro-was originally developed together with Lilly, this collaboration was terminated as we reported in last year's article. While initial data for treatment in people with type 1 and type 2 diabetes (T1D and T2D) have now been published, Adocia is still left without a partner to help get BioChaperone Lispro through critical phase 3 trials. Adocia won its first claim against Eli Lilly \& Company regarding a disputed milestone payment, and additional claims will be assessed in another hearing, but a decision is not expected until 2019 and the future of the program remains unclear at this time.

While the two ultra-long-acting basal insulin analogs, Toujeo (Sanofi) and Tresiba (Novo Nordisk) have now been on the market for several years, we are entering a time during which the efficacy in reducing the risk for hypoglycemia with the ultra-longacting insulins is studied in direct head-to-head randomized controlled trials and also with different methodologies including continuous glucose monitoring. As our review concludes, uncertainty still remains whether there are any clinically meaningful differences between the two at the present time.

The first biosimilar insulin (BioIns) — an insulin glargine developed by Eli Lilly and Boehringer Ingelheim called Abasaglar in the EU and Basaglar in the United States (which was approved in the United States, but not as BioIns) has been on the market now for 1 to 2 years in the EU and the United States. It appears as if this BioIns has gained more market acceptance in the United States than in the EU; it is preferred over the originator insulin glargine in major U.S. formularies, which has induced significant reductions in the sales of the originator insulin. The observed decrease in prices varies between countries, with a maximum of up to $30 \%$. Most likely, a plethora of BioInsparticularly insulin glargines - will be on the market within a few years, which might generate some confusion at the levels of both patients and health-care professionals. As we approach the centennial celebration of insulin discovery, the current scientific progress and controversies around the molecule remain thought-provoking.

\section{Key Articles Reviewed for the Article}

Effect of insulin degludec vs insulin glargine U100 on hypoglycemia in patients with type 1 diabetes. The SWITCH 1 randomized clinical trial

Lane W, Bailey TS, Gerety G, Gumprecht J, Philis-Tsimikas A, Hansen CT, Nielsen TSS, Warren $M$

JAMA 2017; 318: 33-44

Effect of insulin degludec vs insulin glargine U100 on hypoglycemia in patients with type 2 diabetes. The SWITCH 2 randomized trial

Wysham C, Bhargava A, Chaykin L, de la Rosa R, Handelsman $Y$, Troelsen LN, Kvist K, Norwood $P$

JAMA 2017; 318: 45-56 
Day-to-day fasting glycaemic variability in DEVOTE: associations with severe hypoglycaemia and cardiovascular outcomes (DEVOTE 2)

Zinman B, Marso SP, Poulter NP, Emerson SS, Pieber TR, Pratley RE, Lange M, Brown-Frandsen, Moses A, Ocampo Francisco AM, Lekdorf JB, Kvist K, Buse JB; on behalf of the DEVOTE Study Group

Diabetologia 2018; 61: 48-57

DEVOTE 3: temporal relationships between severe hypoglycaemia, cardiovascular outcomes and mortality

Pieber TR, Marso SP, McGuire DK, Zinman B, Poulter NR, Emerson SS, Pratley RE, Woo V, Heller S, Lange M, Brown-Frandsen K, Moses A, Lekdorf JB, Lehmann L, Kvist K, Buse JB; on behalf of the DEVOTE study group

Diabetologia 2018; 61: 58-65

Morning administration of $0.4 \mathrm{U} / \mathrm{kg} / \mathrm{day}$ insulin glargine $300 \mathrm{U} / \mathrm{mL}$ provides less fluctuating 24-hour pharmacodynamic and more even pharmacokinetic profiles compared with insulin degludec $100 \mathrm{U} / \mathrm{mL}$ in type 1 diabetes

Bailey TS, Pettus J, Roussel R, Schmider W, Maroccia M, Nassr N, Klein O, Bolli GB Dahmen $R$

Diabetes Metab 2018; 44: 15-21

Clinical outcomes in real-world patients with type 2 diabetes switching from first- to second-generation basal insulin analogues: comparative effectiveness of insulin glargine 300 units $/ \mathrm{mL}$ and insulin degludec in the DELIVER D + cohort study

Sullivan SD, Bailey TS, Roussel R, Zhou FL, Bosnyak Z, Preblick R, Westerbacka J, Gupta RA Blonde L

Diabetes Obes Metab 2018; 20: 2148-2158

Efficacy and safety of MK-1293 insulin glargine compared with originator insulin glargine (Lantus) in type 1 diabetes: a randomized, open-label clinical trial

Home PD, Lam RLH, Carofano WL, Golm GT, Eldor R, Crutchlow MF, Marcos MC, Rosenstock J, Hollander PA, Gallwitz B

Diabetes Obes Metab 2018; 20: 2220-2228

Efficacy and safety of MK-1293 insulin glargine compared with originator insulin glargine (Lantus) in type 2 diabetes: a randomized, open-label clinical trial

Hollander PA, Carofano WL, Lam RLH, Golm GT, Eldor R, Crutchlow MF, Marcos MC, Rendell $\mathrm{MS}^{3}$, Home PD, Gallwitz B, Rosenstock $J$

Diabetes Obes Metab 2018; 20: 2229-2237

Comparison of morning basal +1 bolus insulin therapy (insulin glulisine + insulin glargine $300 \mathrm{U} / \mathrm{mL}$ vs insulin lispro + insulin glargine biosimilar) using continuous glucose monitoring: a randomized crossover study

Takeishi S, Tsuboi H, Takekoshi S

J Diabetes Investig 2018; 9: 91-99

Efficacy and safety of biosimilar SAR342434 insulin lispro in adults with Type 2 diabetes, also using insulin glargine: SORELLA 2 Study

Derwahl KM, Bailey TS, Wernicke-Panten K, Ping L, Pierre S

Diabetes Technol Ther 2018; 20: 49-58

Safety of insulin lispro and a biosimilar insulin lispro when administered through an insulin pump

Thrasher J, Surks H, Nowotny I, Pierre S, Rotthaeuser B, Wernicke-Panten K, Garg S

J Diabetes Sci Technol 2018; 12: 680-686 
Anti-insulin antibodies and adverse events with biosimilar insulin lispro compared with Humalog insulin lispro in people with diabetes

Home P, Derwahl KM, Ziemen M, Wernicke-Panten K, Pierre S, Kirchhein Y, Garg SK Diabetes Technol Ther 2018; 20: 160-170

Cross-sectional survey of biosimilar insulin utilization in Asia: The Joint Asia Diabetes Evaluation Program

Gani L, Lau E, Luk A, Sobrepena L, Tran QK, Kesavadev J, Jia W, Yu W, Tsang CC, Mukhopadhyay $M$, Jha S, Sheu W, Ho YK, Nguyen TK, Ozaki R, So WY, Kwan C, Fu $A W C$, Mirasol R, Phatak SR, Kumar KMP, Aravind S, Janakiraman H, Chan JCN; on behalf of the JADE Collaborative Study Group

J Diabetes Investig 2018; 9: 1312-1322

Comparative evaluation of pharmacokinetics and pharmacodynamics of insulin glargine (Glaritus ${ }^{\circledR}$ ) and Lantus ${ }^{\circledR}$ : a double-blind, randomized clamp study

Bhatia A, Tawade S, Mastim M, Kitabi EN, Gopalakrishnan M, Shah M, Yeshamaina S, Gobburu J, Sahib M, Thakur D, Prasanna Kumar KM

Acta Diabetol 2018; 55: 461-468

\section{Engineering glucose responsiveness into insulin}

Kaarsholm NC, Lin S, Yan L, Kelly T, van Heek M, Mu J, Wu M, Dai G, Cui Y, Zhu Y, Carballo-Jane E, Reddy V, Zafian P, Huo P, Shi S, Antochshuk V, Ogawa A, Liu F,

Souza SC, Seghezzi W, Duffy JL, Erion M, Nargund RP, Kelley DE

Diabetes 2018; 67: 299-308

Synthetic beta cells for fusion-mediated dynamic insulin secretion

Chen Z, Wang J, Sun W, Archibong E, Kahkoska AR, Zhang X, Lu Y, Ligler FS, Buse JB, Gu Z

Nat Chem Biol 2018; 14: 86-93

Multiresponsive supramolecular theranostic nanoplatform based on pillar[5]arene and diphenylboronic acid derivatives for integrated glucose sensing and insulin delivery

Zuo M, Qian W, Xu Z, Shao W, Hu XY, Zhang D, Jiang J, Sun X, Wang L

Small 2018; 14: e1801942

Core-shell microneedle gel for self-regulated insulin delivery

Wang J, Ye Y, Yu J, Kahkoska AR, Zhang X, Wang C, Sun W, Corder RD, Chen Z, Khan SA, Buse JB, Gu Z

ACS Nano 2018; 12: 2466-2473

Synthetic "smart gel” provides glucose-responsive insulin delivery in diabetic mice

Matsumoto A, Tanaka M, Matsumoto H, Ochi K, Moro-Oka Y, Kuwata H, Yamada H, Shirakawa I, Miyazawa T, Ishii H, Kataoka K, Ogawa Y, Miyahara Y, Suganami $T$

Sci Adv 2017; 3: eaaq0723

Efficacy and safety of rapid-acting insulin analogs in special populations with type 1 diabetes or gestational diabetes: systematic review and meta-analysis

Nørgaard K, Sukumar N, Rafnsson SB, Saravanan $P$

Diabetes Ther 2018; 9: 891-917

A randomized comparison of three prandial insulin dosing algorithms for children and adolescents with type 1 diabetes

Lopez PE, Evans M, King BR, Jones TW, Bell K, McElduff P, Davis EA, Smart CE

Diabet Med 2018; 35: 1440-1447 
Efficacy and safety of fast-acting insulin aspart in comparison with insulin aspart in type 1 diabetes (onset 1): A 52-week, randomized, treat-to-target, phase III trial

Mathieu C, Bode BW, Franek E, Philis-Tsimikas A, Rose L, Graungaard T, Birk Østerskov A, Russell-Jones D

Diabetes Obes Metab 2018; 20: 1148-1155

Investigation of pump compatibility of fast-acting insulin aspart in subjects with type 1 diabetes

Zijlstra E, Demissie M, Graungaard T, Heise T, Nosek L, Bode B

J Diabetes Sci Technol 2018; 12: 145-151

The value of fast-acting insulin aspart compared with insulin aspart for patients with diabetes mellitus treated with bolus insulin from a UK health care system perspective

Leelarathna L, Ashley D, Fidler C, Parekh W

Ther Adv Endocrinol Metab 2018; 9: 187-197

Modeling subcutaneous absorption of fast-acting insulin in type 1 diabetes

Schiavon M, Dalla Man C, Cobelli $C$

IEEE Trans Biomed Eng 2018; 65: 2079-2086

Ultra-rapid BioChaperone lispro improves postprandial blood glucose excursions vs insulin lispro in a 14-day crossover treatment study in people with type 1 diabetes

Andersen $G$, Meiffren $G$, Lamers D, DeVries JH, Ranson A, Seroussi C, Alluis B, Gaudier M, Soula O, Heise T

Diabetes Obes Metab 2018;20: 2627-2632

Greater early postprandial suppression of endogenous glucose production and higher initial glucose disappearance is achieved with fast-acting insulin aspart compared with insulin aspart

Basu A, Pieber TR, Hansen AK, Sach-Friedl S, Erichsen L, Basu R, Haahr H

Diabetes Obes Metab 2018; 20: 1615-1622

\section{ULTRA-LONG-ACTING INSULIN ANALOGS: STILL NO PUBLISHED HEAD-TO-HEAD COMPARISON BETWEEN INSULIN GLARGINE U300 AND INSULIN DEGLUDEC}

Effect of insulin degludec vs insulin glargine U100 on hypoglycemia in patients with type 1 diabetes. The SWITCH 1 randomized clinical trial

Lane $W^{1}$, Bailey $T S^{2}$, Gerety $G^{3}$, Gumprecht $J^{4}$, Philis-Tsimikas $A^{5}$, Hansen $C T^{6}$, Nielsen TSS $^{7}$, Warren $M^{8,9}$

${ }^{1}$ Mountain Diabetes and Endocrine Center, Asheville, NC; ${ }^{2}$ AMCR Institute, Escondido, California; ${ }^{3}$ Albany Medical College, Albany, NY; ${ }^{4}$ Medical University of Silesia, Zabrze, Poland; ${ }^{5}$ Scripps Whittier Diabetes Institute, San Diego, CA; ${ }^{6}$ Medical and Science, Novo Nordisk A/S, Søborg, Denmark; ${ }^{7}$ Biostatistics Insulin and Diabetes Outcomes, Novo Nordisk A/S, Søborg, Denmark; ${ }^{8}$ Physicians East PA, Greenville, NC; ${ }^{9}$ School of Osteopathic Medicine, Campbell University, Lillington, $\mathrm{NC}$

JAMA 2017; 318: 33-44

\section{Background}

The aim of this trial was to assess whether basal insulin supplementation with insulin degludec results in a lower frequency of symptomatic hypoglycemia compared with insulin glargine U100 in adults with type 1 diabetes (T1D).

\section{Methods}

This multicenter, double-blind, randomized crossover noninferiority trial included 501 adults with one or more hypoglycemia risk factors who were surveyed for two consecutive 32-week treatment periods, each consisting of a 16week titration period and a 16-week maintenance period. Participants were randomized to once-daily insulin degludec followed by insulin glargine U100 $(n=249)$ or to the reverse sequence of therapy $(n=252)$. Within each sequence, they were also randomized 1:1 to morning or evening basal insulin administration. The primary outcome was the frequency of overall severe or self-confirmed $(<56 \mathrm{mg} / \mathrm{dL})$ symptomatic hypoglycemic events during the maintenance period. Noninferiority was defined as an upper limit of the two-sided $95 \%$ confidence interval $(95 \% \mathrm{CI})$ for a rate ratio of 1.10 or less; if 
this was the case, a two-sided statistical test for superiority was completed.

\section{Results}

In all, $395(78.8 \%)$ of the randomized participants completed the study. The rates of overall symptomatic hypoglycemia during the maintenance period were 2200.9 events per 100 person-years of exposure (PYE) with insulin degludec and 2462.7 episodes per 100 PYE with insulin glargine U100; the rate ratio (RR) being 0.89 ([95\% CI 0.85 to 0.94]; $P<0.001$ for inferiority; $P<0.001$ for superiority; rate difference -130.31 events per 100 PYE [95\% CI - 193.5 to 67.16]). The occurrence of nocturnal symptomatic hypoglycemia was 277.1 per 100 PYE with insulin degludec and 428.6 per 100 PYE with insulin glargine U100 (RR 0.64 [95\% CI 0.56 to 0.73 ]; $P<0.001$ for noninferiority; $P<0.001$ for superiority; rate difference -61.94 episodes per $100 \mathrm{PYE}$ ([95\% CI -83.85 to -40.03$])$. The proportion of participants experiencing severe hypoglycemic episodes was lower in the insulin degludec group than in the insulin glargine U100 group $(10.3 \%$ [95\% CI $7.3 \%$ to $13.3 \%$ ] vs $17.1 \%$ (95\% CI $13.4 \%$ to $20.8 \%$ ), $P=0.002$; risk difference $-6.8 \%$ (95\% CI $-10.8 \%$ to $-2.7 \%)$.

\section{Conclusions}

In adults with T1D and at least one risk factor for hypoglycemia, treatment for 32 weeks with insulin degludec was associated with lower occurrence of overall symptomatic hypoglycemic events compared with insulin glargine U100.

\section{Effect of insulin degludec vs insulin glargine U100 on hypoglycemia in patients with type 2 diabetes. The SWITCH 2 randomized trial}

Wysham $C^{1}$, Bhargava $A^{2}$, Chaykin $L^{3}$, de la Rosa $R^{4}$, Handelsman $Y^{5}$, Troelsen $L N^{6}$, Kvist $K^{7}$, Norwood $P^{8}$

${ }^{1}$ Rockwood Clinic. University of Washington School of Medicine, Spokane, WA; ${ }^{2}$ Iowa Diabetes and Endocrinology Research Center, Des Moines, IA; ${ }^{3}$ Meridien Research, Bradenton, FL; ${ }^{4}$ Paducah Endocrinology, Paducah, KY; ${ }^{5}$ Metabolic Institute of America, Tarzana, CA; ${ }^{6}$ Medical \& Science, Novo Nordisk A/S, Søborg, Denmark; ${ }^{7}$ Biostatistics Insulin \& Diabetes Outcomes, Novo Nordisk A/S, Søborg, Denmark; ${ }^{8}$ Valley Research, Fresno, CA

JAMA 2017; 318: 45-56

\section{Background}

The aim of this study was to evaluate whether basal insulin therapy with insulin degludec is associated with less exposure to hypoglycemia compared with insulin glargine U100 in adults with type 2 diabetes.

\section{Methods}

A multicenter, double-blind, treat-to-target crossover trial was conducted over two consecutive 32 -week periods (16 weeks for titration and 16 weeks of maintenance). Adult subjects $(n=721)$ with type 2 diabetes (T2D) and one or more hypoglycemia risk factors treated with basal insulin with or without oral antidiabetic drugs were randomized to receive once-daily insulin degludec followed by insulin glargine $\mathrm{U} 100(\mathrm{n}=361)$ or the reverse order $(\mathrm{n}=360)$, and randomly assigned (1:1) to morning or evening dosing within each sequence period.

\section{Results}

A total of 580 of the 721 randomized participants (80.4\%) finished the trial. Overall symptomatic hypoglycemic events (severe hypoglycemia or confirmed blood glucose $<56 \mathrm{mg}$ / $\mathrm{dL}$ ) during the maintenance period (primary outcome) occurred at rates of 185.6 and 265.4 episodes per 100 patientyears of exposure (PYE) in the degludec and glargine U100 groups, respectively (rate ratio (RR) 0.70 [95\% CI $0.61-$ 0.80]; $P<0.001$; rate difference -23.66 events/100 PYE [95\% CI -33.98 to -13.33$])$. The percentage of participants with registered hypoglycemic events was $22.5 \%$ using degludec and $31.6 \%$ using glargine U100 (difference $-9.1 \%$ [95\% CI $-13.1 \%$ to $-5.0 \%]$ ). Rates of nocturnal hypoglycemic events with insulin degludec vs glargine U100 were 55.2 and 93.6 episodes/100 PYE, respectively (RR 0.58 [95\% CI 0.46-0.74], $P<0.001$; difference -7.41 events/100 PYE [95\% CI -11.98 to -2.85$]$ ), and the relative proportions of participants with nocturnal hypoglycemia were $9.7 \%$ vs $14.7 \%$ (difference $-5.1 \%$ [95\% CI $-8.1 \%$ to $-2.0 \%]$ ). Patients experienced severe hypoglycemic events during the maintenance period at a rate of $1.6 \%$ [95\% CI $0.6 \%-2.7 \%$ ] in the degludec group and $2.4 \%$ [96\% CI 1.1\%-3.7\%] in the glargine U100 group $(P=0.35$; risk difference $-0.8 \%[95 \%$ CI $-2.2 \%$ to $0.5 \%])$.

\section{Conclusions}

In adults with T2D with at least one risk factor for hypoglycemia using basal insulin therapy, treatment for 32 weeks with insulin degludec resulted in lower frequency of overall symptomatic hypoglycemia compared with insulin glargine U100.

\section{Day-to-day fasting glycaemic variability in DEVOTE: associations with severe hypoglycaemia and cardiovascular outcomes (DEVOTE 2)}

Zinman $B^{1}$, Marso $S P^{2}$, Poulter $N^{3}$, Emerson $S S^{4}$, Pieber $T R^{5}$, Pratley $R E^{6,7}$, Lange $M^{8}$, Brown-Frandsen ${ }^{8}$, Moses $A^{8}$, Ocampo Francisco $A M^{8}$, Lekdorf $\mathrm{JB}^{8}$, Kvist $K^{8}$, Buse $\mathrm{JB}^{9}$; on behalf of the DEVOTE Study Group

${ }^{1}$ Lunenfeld-Tanenbaum Research Institute, Mount Sinai Hospital, University of Toronto, Toronto, Canada; ${ }^{2} \mathrm{Re}-$ search Medical Center, Kansas City, MO; ${ }^{3}$ Imperial Clinical Trials Unit, Imperial College, London, UK; ${ }^{4}$ University of Washington, Seattle, WA; ${ }^{5}$ Medical University of Graz, Graz, Austria; ${ }^{6}$ Florida Hospital Translational Research Institute for Metabolism and Diabetes, Orlando, FL; ${ }^{7}$ Sanford Burnham Prebys Medical Discovery Institute, Orlando, FL; ${ }^{8}$ Novo Nordisk A/S, S $\phi$ borg, Denmark; ${ }^{9}$ University of North Carolina School of Medicine, Chapel Hill, NC

Diabetologia 2018; 61: 48-57

\section{Background}

The Trial Comparing Cardiovascular Safety of Insulin Degludec vs Insulin Glargine in Patients With Type 2 
Diabetes at High Risk of Cardiovascular Events (DEVOTE) trial assessed the cardiovascular safety of insulin degludec vs insulin glargine U100 in adults with type 2 diabetes at high risk of cardiovascular events and showed that insulin degludec was noninferior to glargine U100 in terms of major adverse cardiovascular events (MACE) and superior with regard to occurrence of both overall and nocturnal severe hypoglycemia. The present study was a secondary analysis using data from 7586 participants over a median observation time of 1.99 years to evaluate the relationships between day-to-day fasting glycemic variability vs severe hypoglycemia and MACE.

\section{Methods}

The day-to-day fasting variability was calculated based on patients' self-monitored blood glucose (SMBG) recordings before breakfast, performed 3 days before each monthly study site contact. The variance of the three log-transformed SMBG recordings was determined for each patient, and standard deviation was calculated as the square root of the mean of these monthly variances as a measure of day-to-day fasting glycemic variability. Associations between fasting glycemic variability and severe hypoglycemia, MACE, and all-cause mortality were analyzed with Cox proportional hazards models.

\section{Results}

In nonadjusted models, day-to-day fasting glycemic variability was significantly associated with severe hypoglycemia (hazard ratio 4.11 [95\% CI 3.15-5.35]), MACE (HR 1.36 [95\% CI 1.12-1.65]), and all-cause mortality (HR 1.58 [95\% CI 1.23-2.03]). Relationships between day-to-day fasting glycemic variability vs severe hypoglycemia and all-cause mortality remained significant after various adjustments, whereas the significant association with MACE was not maintained after adjustment for baseline glycated hemoglobin (HbA1c) (HR 1.19 [95\% CI 0.96-1.47]) or last HbA1c during the trial period (HR 1.21 [95\% CI 0.98-1.49]).

\section{Conclusions}

The data indicate that higher day-to-day fasting glycemic variability is associated with increased risks of severe hypoglycemia and all-cause mortality.

\section{DEVOTE 3: temporal relationships between severe hypoglycaemia, cardiovascular outcomes and mortality}

Pieber $T R^{1}$, Marso $S P^{2}$, McGuire $D K^{3}$, Zinman $B^{4}$, Poulter $\mathrm{NR}^{5}$, Emerson $S S^{6}$, Pratley $R E^{7,8}$, Woo $V^{9}$, Heller $S^{10}$, Lange $M^{11}$, Brown-Frandsen $K^{11}$, Moses $A^{11}$, Lekdorf $\mathrm{JB}^{11}$, Lehmann $L^{11}$, Kvist $K^{11}$, Buse $\mathrm{JB}^{12}$; on behalf of the DEVOTE study group

${ }^{1}$ Medical University of Graz, Graz, Austria; ${ }^{2}$ Research Medical Center, Kansas City, MO; ${ }^{3}$ University of Texas Southwestern Medical Center, Dallas, TX; ${ }^{4}$ LunenfeldTanenbaum Research Institute, Mount Sinai Hospital, University of Toronto, ON, Toronto, Canada; ${ }^{5}$ Imperial Clinical Trials Unit, Imperial College, London, UK; ${ }^{6}$ University of Washington, Seattle, WA; ${ }^{7}$ Florida Hospital Translational Research Institute for Metabolism and Diabetes, Orlando, FL; ${ }^{8}$ Sanford Burnham Prebys Medical Discovery Institute,
Orlando, FL; ${ }^{9}$ University of Manitoba, Winnipeg, $\mathrm{MB}, \mathrm{Ca}$ nada; ${ }^{10}$ Academic Unit of Diabetes, Endocrinology and Metabolism, University of Sheffield, Sheffield, UK; ${ }^{11}$ Novo Nordisk A/S, Sфborg, Denmark; ${ }^{12}$ University of North Carolina School of Medicine, Chapel Hill, NC

\section{Diabetologia 2018; 61: 58-65}

\section{Background}

This secondary analysis of the DEVOTE trial aimed to investigate associations between severe hypoglycemia vs cardiovascular events and all-cause mortality.

\section{Methods}

Associations between severe hypoglycemia and MACE and all-cause mortality were assessed by comparing the risk of an event with or without a prior severe hypoglycemic episode in different time periods, using Cox regression models. As an analysis with interaction terms showed that the impact of severe hypoglycemia on MACE and all-cause mortality was similar for the two treatment arms with insulin degludec and glargine U100, the analyses were performed using the pooled trial population.

\section{Results}

A significantly higher risk of all-cause mortality was found for participants who had experienced severe hypoglycemia vs those who had not for the whole trial observation period (HR 2.51 [95\% CI 1.79-3.50]), as well as during specific time intervals following severe hypoglycemia; the risk being highest early on after the severe hypoglycemic event, but still significant at one year thereafter. The association between severe hypoglycemia and all-cause mortality was also maintained after adjustment for various baseline parameters. Conversely, there was no significant difference regarding the risk of MACE, whether severe hypoglycemia had been experienced or not (HR 1.38 [95\% CI 0.96-1.96]; $P=0.080)$, and hence no temporal relationship between severe hypoglycemia and MACE.

\section{Conclusions}

The findings demonstrate an association between severe hypoglycemia and all-cause mortality, with the greatest risk in the short term after a severe hypoglycemic event. Whether severe hypoglycemia is merely a marker for increased mortality risk or mediates a direct causal effect cannot be elucidated with certainty from the observed associations.

\section{Morning administration of $0.4 \mathrm{U} / \mathrm{kg} / \mathrm{day}$ insulin glargine $300 \mathrm{U} / \mathrm{mL}$ provides less fluctuating 24-hour pharmacodynamic and more even pharmacokinetic profiles compared with insulin degludec $100 \mathrm{U} / \mathrm{mL}$ in type 1 diabetes}

Bailey $T S^{1}$, Pettus $J^{2}$, Roussel $R^{3,4,5}$, Schmider $W^{6}$, Maroccia $M^{7}$, Nassr $N^{6}$, Klein $O^{8}$, Bolli $G B^{9}$ Dahmen $R^{6}$

${ }^{1}$ AMCR Institute, Escondido, CA; ${ }^{2}$ University of California, San Diego, CA; ${ }^{3}$ Inserm U1138, Centre de Recherche des Cordeliers, Paris, France; ${ }^{4}$ Université Paris Diderot, Sorbonne Paris Cité, Paris, France; ${ }^{5}$ Diabetology, Endocrinology 
and Nutrition Department, DHU FIRE Hôpital Bichat, Paris, France; ${ }^{6}$ Sanofi-Aventis Deutschland GmbH, Frankfurt am Main, Germany; ${ }^{7}$ Umanis, Levallois-Perret, France; ${ }^{8}$ Profil Neuss, Germany; ${ }^{9}$ Department of Medicine, University of Perugia Medical School, Perugia, Italy

Diabetes Metab 2018; 44: 15-21

\section{Background}

The aim of this study was to evaluate steady-state pharmacodynamics (PD) and pharmacokinetic (PK) profiles of insulin glargine U300 vs insulin degludec $100 \mathrm{U} / \mathrm{mL}$ in adults with T1D.

\section{Methods}

Participants in this randomized, double-blind, crossover euglycemic clamp trial received either $0.4 \mathrm{U} / \mathrm{kg}(\mathrm{n}=24)$ or $0.6 \mathrm{U} / \mathrm{kg}(\mathrm{n}=24)$ once-daily (before breakfast) basal insulin administration for 8 days and were randomized to start with insulin glargine U300 or insulin degludec. Thereafter, and after a wash-out period for 8-26 days, they received the opposite insulin treatment. Euglycemic clamp procedures were done for up to 30 hours after basal insulin administration on day 8 in each treatment sequence. The primary outcome was within-day variability of the smoothed glucose infusion rate (GIR) profile over 24 hours.

\section{Results}

With $0.4 \mathrm{U} / \mathrm{kg} / \mathrm{day}, 24 \mathrm{~h}$ steady-state smoothed GIR profile variability (daily fluctuation) was $20 \%$ less with insulin glargine U300 than with insulin degludec (treatment ratio 0.80 [90\% CI 0.66-0.96], $P=0.047$ ), whereas with $0.6 \mathrm{U} / \mathrm{kg} /$ day it was similar between the two basal insulins (treatment ratio 0.96 [90\% CI $0.83-1.11], P=0.603$ ). Both insulins displayed exposure and efficacy until the end of the clamp period $(30 \mathrm{~h})$, but the PK profiles appeared more evenly distributed with glargine U300 than with degludec at both the $0.4 \mathrm{U} / \mathrm{kg}$ and $0.6 \mathrm{U} / \mathrm{kg}$ dose levels.

\section{Conclusion}

Insulin glargine U300 displays less fluctuating steady-state PD profiles (lower within-day variability) and more even PK profiles compared with insulin degludec, when basal insulin is given in a once-daily, morning dosing regimen of $0.4 \mathrm{U} / \mathrm{kg} /$ day.

\section{Clinical outcomes in real-world patients with type 2 diabetes switching from first- to second-generation basal insulin analogues: comparative effectiveness of insulin glargine 300 units $/ \mathrm{mL}$ and insulin degludec in the DELIVER $\mathrm{D}+$ cohort study}

Sullivan $S D^{1}$, Bailey $T S^{2}$, Roussel $R^{3,4,5}$, Zhou $F L^{6}$, Bosnyak $Z^{7}$, Preblick $R^{6}$, Westerbacka $J^{7}$, Gupta $R A^{8}$ Blonde $L^{9}$

${ }^{I}$ School of Pharmacy, University of Washington, Seattle, WA; ${ }^{2}$ AMCR Institute, Escondido, CA; ${ }^{3}$ Inserm U1138, Centre de Recherche des Cordeliers, Paris, France; ${ }^{4}$ Université Paris Diderot, Sorbonne Paris Cité, Paris, France; ${ }^{5}$ Diabetology, Endocrinology and Nutrition Department, DHU FIRE, Hôpital Bichat, AP-HP, Paris, France; ${ }^{6}$ Sanofi, Bridgewater, New Jersey; ${ }^{7}$ Sanofi, Paris, France; ${ }^{8}$ Accent- ure, Florham Park, NJ; ${ }^{9}$ Frank Riddick Diabetes Institute, Endocrinology Department, Ochsner Medical Center, New Orleans, LA

Diabetes Obes Metab 2018; 20: 2148-2158

\section{Background}

The aim of this real-world study was to assess the clinical outcomes in adults with T2D after switching from insulin glargine 100 units $/ \mathrm{mL}$ or insulin detemir to insulin glargine 300 units/mL or insulin degludec.

\section{Methods}

A retrospective, observational study of electronic medical records (EMR) was carried out for adults who switched to insulin glargine U300 or degludec between March 1, 2015 to January 31, 2017, with data records for at least 12 months before the switch, including at minimum a valid measurement of HbA1c during the last 6-month period and a followup period of at least 6 months thereafter. Those who switched to glargine U300 or to degludec were propensity-score matched based on baseline demographic or clinical characteristics. Study outcomes were change in glycemic control and goal attainment (HbA1c) as well as incidence of hypoglycemia (based on ICD-9/ICD-10 diagnoses or events of blood glucose $\leq 3.9 \mathrm{mmol} / \mathrm{L}$ reported in the EMRs, including those related to emergency department visits or hospitalization).

\section{Results}

Each matched cohort consisted of 1592 adults. The mean reduction of $\mathrm{HbA1c}$ and attainment rates of $\mathrm{HbA} 1 \mathrm{c}$ goals below $7.0 \%(53 \mathrm{mmol} / \mathrm{mol})$ or $8.0 \%(64 \mathrm{mmol} / \mathrm{mol})$ were comparable in glargine U300- and degludec-switchers (data available for 742 vs 727 patients respectively). When assessed with fixed follow-up at 6 months (intention-to-treat cohorts), the proportion of patients experiencing overall hypoglycemic events was lowered from $15.6 \%$ to $12.7 \%(P=0.006)$, and hypoglycemia-caused emergency department or in-patient care from $5.3 \%$ to $3.5 \%(P=0.007)$ after the switch to glargine U300; no significant changes were registered after switching to insulin degludec. After adjusting for baseline hypoglycemia, the incidence and relative event rate of hypoglycemia were comparable for glargine U300 vs degludec. Using variable follow-up (full 6-month follow-up period or to discontinuation of therapy if this occurred earlier), the incidence of all hypoglycemic events was similar in both cohorts, but the inpatient/ emergency department event rate was significantly lower with glargine U300 than with degludec (event ratio $0.56 ; P=0.016$ ).

\section{Conclusions}

In real-world clinical practice, the change of basal insulin therapy to either glargine U300 or degludec in adults with type 2 diabetes resulted in similar improvements in glycemic control and reductions in hypoglycemic events.

\section{Comments}

While the two ultra-long-acting basal insulin analogs, insulin degludec (Tresiba) and insulin glargine U300 
(Toujeo), now have been on the market for several years, no direct head-to-head randomized controlled trials have hitherto been published, and thus uncertainty still remains whether there are any clinically meaningful differences between the two. As has been comprehensively reviewed in earlier yearbooks, many trials have been conducted in which either Tresiba or Toujeo were compared with first-generation long-acting analogs (in most cases insulin glargine U100 and less often insulin detemir), showing reduced risk of hypoglycemia-especially nocturnal hypoglycemia—as a consequence of their protracted and less variable pharmacokinetic and pharmacodynamics profiles. With insulin degludec, this was again corroborated in the two referenced SWITCH trials in T1D and T2D, with glargine U100 being the comparator in both. The novelty of the later studies compared with earlier ones was, first, the cross-over design in which all participants received both treatments in random order - and hence served as their own controls with a wash-out period between the crossovers to minimize any carryover effects, and secondly, that only adults with established risk factors for hypoglycemia were included. The definition of hypoglycemia used in the studies (rate of overall severe or symptomatic, blood-glucose-confirmed $<56 \mathrm{mg} / \mathrm{dL}$ hypoglycemia), however, may render comparisons of the incidence rates of hypoglycemia with findings in other trials somewhat difficult. Nevertheless, as hypoglycemia constitutes the main obstacle to attain glycemic treatment goals, a lowering of this risk definitely constitutes a therapeutic advance. The referenced secondary analyses with data from the degludec cardiovascular outcomes study, the DEVOTE trial (1), also add to the accumulating evidence of detrimental interrelated associations between high glycemic variability, severe hypoglycemia, cardiovascular events, and mortality.

With regard to published data on comparisons of the pharmacodynamics of insulin degludec and glargine U300, the picture is also slightly unclear. Last year we reported on a comprehensive cross-over, euglycemic clamp study be Heise et al. (2). This Novo Nordiskinitiated study demonstrated lower day-to-day and within-day variability and a more stable glucoselowering efficacy for degludec vs glargine U300. Conversely, in the presently referenced study by Bailey and co-workers, which was supported by Sanofi and conducted at the same research center as the former, smaller within-day variability was demonstrated with glargine U300 than with degludec. These seemingly contradictive results have caused a debate about methodological confounders associated with clamp experiments (such as insulin dosing, timing of insulin administration, differences in calculating within-day variability, and curve smoothing procedures) that might influence the assessment of pharmacodynamic parameters (3-6). Obviously, there is a need for standardization for assessing pharmacodynamic characteristics, to allow confirmed comparisons between different ultra-longacting insulins. Then, again the question remains whether subtle differences in these assessments will have any major clinical significance. From the retrospective "real-world" analysis by Sullivan and coworkers, it seems that the efficacy of insulin degludec and glargine U300 in terms of glucose control and alleviating the risk of hypoglycemia in adults with type 2 diabetes is comparable. Preliminary findings from a randomized, controlled, head-to-head comparison between the two insulin analogs (7) speak in the same direction. Hopefully, we will be able to comment in more detail on the complete results of the trial (and perhaps others) in the next yearbook.

\section{BIOSIMILAR INSULIN}

Only one study reporting the results of glucose clamp studies in Asian healthy subjects with a BioIns was published in 2017 (8). No significant differences in PK and PD properties in comparison to the originator insulin glargine were observed.

Once more insulins are approved as BioIns and can be launched (see below), they are entering a market with wellestablished players that are fighting for their market shares. Gaining a market share will mainly be possible by providing additional cost reductions or by means of political pressure. If the price of insulin is decreasing too much (as with test strips), this can in turn reduce the interest of companies in investing in research for new insulins, etc. If insulin were to become generic, it might even cause a decrease in supply by many companies (they will step out if the business is not attractive), as it has been seen recently with other drugs.

Currently > 20 companies have BioIns in clinical development, not only for insulin glargine, but also for other insulin analogs and human insulin formulations. It should be kept in mind that even small differences in the structure of the insulin molecules manufactured ("micro-heterogeneity") can have an impact on its metabolic effect. It will remain to be seen how many BioIns will come to the market at the end.

\section{Approved biosimilar insulin on the market: Admelog}

More recently the first BioIns (Admelog) of a rapid-acting insulin analog (insulin lispro), developed by Sanofi, was approved and is now on the market in the United States and the European Union. Articles from both Derwahl and colleagues and P. Home and colleagues report results from recent studies of this BioIns in patients with T1D or T2D and it is on the market on both continents now. In addition, two articles-one from P. Home and colleagues presenting data on the risk of developing insulin antibodies and one from Thrasher and colleagues examining the use of this BioIns in insulin pumps - show no difference in the properties of this BioIns compared with the originator insulin.

\section{Approved biosimilar insulins not on the market: Lusduna}

The next approved BioIns most likely to come to the market in the EU and the United States is another insulin glargine developed by Merck-U.S. (Lusduna). Merck refrained from launching this BioIns, as they are blocked by a lawsuit against them by Sanofi. In the past, Eli Lilly was able 
to get a settlement deal with Sanofi for Basaglar. However, the recent publications from P.D. Home et al. and from Hollander et al. (discussed here) present the results of clinical trials for Merck's insulin glargine in patients with T1D or T2D. Data from two glucose clamp studies with this BioIns have also been published (9).

\section{Not-yet-approved Biolns in the United States but approved in the EU: Semglee}

Semglee is an insulin glargine that the U.S.-based generics giant Mylan has developed in cooperation with the huge Indian pharmaceutical company BioCon. This BioIns was approved in the EU recently; however, it is not clear when and if it will come to the market in the EU (plans are for the end of 2018, second to the market if Lusduna is not launched before), since EU inspectors raised concerns about the production facility in India. Inspectors from the U.S. FDA also raised serious concerns. However, both companies are confident that they can solve the issues and that they are progressing according to their plans for the launch of this BioIns in the United States (plans set are for 2020). It should be mentioned that this BioIns is already on the market in Japan, South Korea, and the United Arab Emirates.

Mylan filed an application to the FDA for their insulin glargine in prefilled pen and vial forms under the 505(b)(2) regulatory pathway. The pathway enables a faster regulatory approval process, relying partly on data already generated by a previous drug maker. They are also seeking an interchangeability designation (see below).

Sanofi is not willing to give up rights on its originator insulin glargine, which is a billion-dollar drug for them. Thus, Sanofi filed a lawsuit against Mylan in the United States alleging infringement of its patents.

\section{Prices}

As stated, until now market introduction of BioIns has not induced drastic changes in the insulin market; however, when more BioIns enter the market, the increased competition will probably change this rapidly. The hot debate about insulin prices in the United States-which have gone up massively in recent years-might affect this as well. Another question is whether one of the BioIns will get approval (e.g., by the FDA) for interchangeability (see below). It must also be acknowledged that when it comes to the cost of insulin therapy, each country has his own complex reimbursement system for drugs in place, making it difficult to compare the situation in one country with that in another country. The global insulin market is $>\$ 40$ billion (U.S.), and the final price of insulin depends on many factors-pharmaceutical companies are only one player.

\section{Immunological aspects}

A matter of concern is whether BioIns might differ in their immunogenic properties from the originator insulin. The studies required during the approval process, a clinical study with a limited number of subjects (300) over a limited period of time (6/12 months), might not be able to clarify if such reactions do show up in certain patient groups after a prolonged period of usage. A major concern is that, at least in some patients, formation of (neutralizing) insulin antibodies is stimulated. According to the study from P. Home et al., differences in immunological responses might be induced by certain differences between the BioIns and the originator insulin [see also (10)].

\section{Quality}

In the same line of thinking, it would be of interest to know the quality of different batches of (originator) insulins and BioIns is over time. Insulin is manufactured in batches and these might differ considerably from each other as a reflection of the complexity of the manufacturing process. The amount of information that companies are reporting about this is quite limited $(11,12)$.

\section{Interchangeability}

Switching from one insulin to another is a decision made by the treating physician, to exchange one insulin with another that has the same therapeutic intent in a given patient (13). Interchangeability means the practice of substituting one insulin for another that is expected to achieve the same blood glucose-lowering effect in any patient with diabetes on the initiative or with the agreement of the physician. This requires that the insulin can be regarded as interchangeable (i.e., as a property of a given insulin). The FDA has relatively lately characterized what their expectations are for such a label; however, until now no BioIns has received such a label. In contrast, substitution is a process in which-at the pharmacy level without consulting the prescribing physiciananother insulin (a BioIns) is dispensed instead of the prescribed insulin. Currently there is no "substitutability determination" in the EU; that is, there is no automatic substitution in practice in the EU whereby a pharmacist is obliged to dispense a BioIns instead of a prescribed insulin. Interesting differences exist between states in the United States and also within the EU when it comes to guidelines for market allowance of BioIns.

\section{Devices}

In daily practice for patients, diabetologists, and nurses, the devices (typically insulin pens) used for application of insulin are of high relevance (14). Each manufacturer of BioIns comes to the market with a combination of, for example, their insulin glargine with an insulin pen, which, as indicated, is associated with a certain risk of confusion. The switch from the pen(s) of the originator insulin to those of another insulin is associated with an additional teaching and training burden for the diabetes team. Such aspects are of high relevance from a practical point of view.

\section{Reviews, systematic reviews, and meta-analysis}

Once the first reviews, systematic reviews, and metaanalysis about a given topic are published, this can be regarded as a sign of a certain maturity in this area of research. One review reflected mainly on the introduction of BioIns in the EU (15). A U.S. team recently published a systematic review of five different BioIns for which studies have shown no difference between the BioIns and the originator insulins (16). It is of interest to note that the 11 randomized controlled trials (dealing mainly with BioIns of insulin glargine and insulin lispro only) analyzed were regarded as being of 
moderate quality. However, the study data suggest similar clinical efficacy and safety of these BioIns. In a second systematic review and meta-analysis by a Japanese group of researchers, 10 trials were assessed (two trials each on four BioIns, and one trial each on two others) (17). This metaanalysis found no differences between long-acting BioIns and originator insulins with regard to reduction in glucose control (i.e., improvement in HbA1c) or reduction in fasting plasma glucose, mortality, injection site reactions, insulin antibodies, or allergic reactions. This meta-analysis also showed no significant differences in clinical efficacy and safety, including immune reactions, between BioIns and originator insulins.

\section{Summary}

These are still early days for BioIns. Those that are currently approved in the United States were approved by a 505(b)(2) abbreviated pathway by the FDA; future BioIns are likely to be regulated through the $351(\mathrm{k})$ pathway, which was designed specifically for biosimilars. Will have to see if any changes show up in the United States when this approval process for insulins becomes active in 2020.

One can reasonably assume that BioIns will gain more attention and momentum in the next years. Cost aspects will mainly drive this. It is also of interest to note that neither the American Diabetes Association nor the European Association for the Study of Diabetes has issued any position paper about BioIns yet. When the BioIns market expands, more questions will emerge regarding the safety, effectiveness, and interchangeability of BioIns and their reference products. Relative to how commonly these products are used, little scientific evidence exists regarding these issues.

\section{Efficacy and safety of MK-1293 insulin glargine compared with originator insulin glargine (Lantus) in type 1 diabetes: a randomized, open-label clinical trial}

Home $P D^{1}$, Lam $R L H^{2}$, Carofano $W L^{2}$, Golm $G T^{2}$, Eldor $R^{2}$, Crutchlow $M F^{2}$, Marcos $M C^{2}$, Rosenstock $\mathrm{J}^{3}$, Hollander $\mathrm{PA}^{4}$, Gallwitz $B^{5}$

${ }^{1}$ Newcastle University, Newcastle upon Tyne, UK; ${ }^{2}$ Merck \& Co., Inc., Kenilworth, NJ; ${ }^{3}$ Dallas Diabetes Research Center at Medical City, Dallas, TX; ${ }^{4}$ Baylor Endocrine Center, Dallas, TX; ${ }^{5}$ University of Tübingen, Tübingen, Germany

Diabetes Obes Metab 2018; 20: 2220-2228

\section{Background}

The efficacy and safety of an insulin glargine developed by Merck (Mk-Gla; $100 \mathrm{U} / \mathrm{mL}$ ) was compared with that of insulin glargine manufactured by the originator (Sanofi Sa-Gla) in patients with type 1 diabetes mellitus (T1D).

\section{Methods}

Five hundred and eight individuals with T1D (HbA1c $\leq 11.0 \%$; $97 \mathrm{mmol} / \mathrm{mol}$ ) taking basal and prandial insulin were enrolled in this phase 3, randomized, active-controlled, open-label, 52-week study. They were randomized 1:1 to once-daily Mk-Gla $(n=245)$ or Sa-Gla $(n=263)$. Based on a prebreakfast plasma glucose dosing algorithm dose titration of basal insulin was performed. Primary efficacy objective was assessment of the noninferiority of $\mathrm{HbA} 1 \mathrm{c}$ change from baseline (margin of $0.4 \%$ [ $4.4 \mathrm{mmol} / \mathrm{mol}]$ ) for both glargines over 24 weeks. Primary safety objective was assessment of anti-insulin antibody development over 24 weeks.

\section{Results}

Change in mean $\mathrm{HbA} 1 \mathrm{c}$ from baseline at week 24 was $0.62 \%(95 \%$ CI $-0.79 \%$ to $-0.45 \%)$ for Mk-Gla and $0.66 \%$ (CI $-0.82 \%$ to $-0.50 \%$ ) for Sa-Gla. Mean HbA1c difference was $0.04 \%$ (CI $-0.11 \%$ to $0.19 \%$ ) for Mk-Gla without Sa-Gla, meeting the primary and secondary objective criteria for noninferiority and equivalence. Mean insulin glargine dose for both glargines was 0.46 and $0.48 \mathrm{U} / \mathrm{kg}$ at week 24. Similarity of $\mathrm{HbA} 1 \mathrm{c}$ response and basal insulin dose trajectory persisted over the study period. Also, safety and tolerability, including anti-insulin antibody responses, hypoglycemia, adverse events, and body weight, were similar between both glargines during the study period.

\section{Conclusions}

Both glargines exhibited similar efficacy and safety in patients with T1DM.

\section{Efficacy and safety of MK-1293 insulin glargine compared with originator insulin glargine (Lantus) in type 2 diabetes: a randomized, open-label clinical trial}

Hollander $P A^{1}$, Carofano $W L^{2}$, Lam $R L H^{2}$, Golm $G T^{2}$, Eldor $R^{2}$, Crutchlow $M F^{2}$, Marcos $M C^{2}$, Rendell $\mathrm{MS}^{3}$, Home $P D^{4}$, Gallwitz $B^{5}$, Rosenstock $J^{6}$

${ }^{1}$ Baylor Endocrine Center, Dallas, TX; ${ }^{2}$ Merck \& Co., Inc., Kenilworth, NJ; ${ }^{3}$ The Rose Salter Medical Research Foundation, Newport Coast, CA; ${ }^{4}$ Newcastle University, Newcastle upon Tyne, UK; ${ }^{5}$ University of Tübingen, Tübingen, Germany; ${ }^{6}$ Dallas Diabetes Research Center at Medical City, Dallas, TX

Diabetes Obes Metab 2018; 20: 2229-2237

\section{Background}

The efficacy and safety of an insulin glargine developed by Merck (Mk-Gla; $100 \mathrm{U} / \mathrm{mL}$ ) was compared with that of insulin glargine manufactured by the originator (Sanofi Sa-Gla) in patients with type 2 diabetes mellitus (T2DM).

\section{Methods}

Five hundred and thirty-one people with T2DM (HbA1c $\leq 11.0 \% ; 97 \mathrm{mmol} / \mathrm{mol}$ ) and eligible for or currently taking basal insulin ( $\geq 10 \mathrm{U} /$ day) were enrolled in this phase 3, randomized, active-controlled, open-label, 52-week study. Participants were randomized 1:1 to once-daily Mk-Gla $(n=263)$ or Sa-Gla $(n=263)$. Dose titration of basal insulin was based on a fasting plasma glucose (FPG) dosing algorithm. The primary efficacy objective was assessment of the noninferiority of $\mathrm{HbAlc}$ change from baseline (margin of $0.4 \%$; $4.4 \mathrm{mmol} / \mathrm{mol}$ ) for both glargines over 24 weeks. Assessment of anti-insulin antibody development over 24 weeks was the primary safety objective. 


\section{Results}

Change in mean $\mathrm{HbAlc}$ from baseline at week 24 was $1.28 \%(95 \% \mathrm{CI}-1.41 \%$ to $-1.15 \%)$ and $-1.30 \%(-1.43 \%$ to $-1.18 \%$ ) for Mk-Gla and Sa-Gla. Mean HbA1c difference was $0.03 \%(-0.12 \%$ to $0.18 \%)$ for Mk-Gla minus Sa-Gla, meeting the primary and secondary objective criteria for noninferiority and equivalence. Insulin doses, FPG, and seven-point plasma glucose profiles were similar between groups. Also, safety and tolerability, including anti-insulin antibody responses, hypoglycemia, adverse events, and body weight, were similar across both glargines during the study period. The efficacy and safety of both glargines were similar both in participants who were insulin treated or insulin naïve at baseline.

\section{Conclusions}

Both glargines exhibited similar efficacy and safety in patients with T2DM.

\section{Comparison of morning basal +1 bolus insulin therapy (insulin glulisine + insulin glargine $300 \mathrm{U} /$ $\mathrm{mL}$ vs insulin lispro + insulin glargine biosimilar) using continuous glucose monitoring: a randomized crossover study}

Takeishi S, Tsuboi H, Takekoshi S

Department of Diabetes, General Inuyamachuo Hospital, Inuyama-city, Aichi, Japan

J Diabetes Investig 2018; 9: 91-99

\section{Background}

The effects of morning administration of insulin glulisine + insulin glargine $300 \mathrm{U} / \mathrm{mL}(\mathrm{G}+\mathrm{G} 300)$ with that of insulin lispro + insulin glargine biosimilar $(\mathrm{L}+\mathrm{GB})$ were compared in a clinical study.

\section{Materials and Methods}

Thirty patients with type 2 diabetes who used a continuous glucose monitoring system on study admission after glucose levels were stabilized by subcutaneous injections of long-acting and ultra-rapid-acting insulins (before test meals were given) on days 1 and 2, and the same dose $\mathrm{L}+\mathrm{GB}$ on days 3 and 4 , or vice versa. Allocation to the groups was done randomly. Mean amplitude of glycemic excursion and mean of daily differences were analyzed. Insulin was injected at 8:00 a.m. A day was defined as the period from $08.00 \mathrm{~h}$ one day to $08.00 \mathrm{~h}$ the next day.

\section{Results}

In patients taking $\mathrm{G}+\mathrm{G} 300$, postbreakfast glucose level, postbreakfast glucose gradient, mean glucose level, standard deviation, and M-value (24 h, 00:00-06:00 h), mean amplitude of glycemic excursion, and mean of daily differences were significantly lower than in individuals taking $\mathrm{L}+\mathrm{GB}$ $(P \leq 0.0001-0.04)$. Area over the glucose curve $(<70 \mathrm{mg} /$ $\mathrm{dL}$ ), however, was not different between groups. Nevertheless, glucose levels were lower in patients taking L + GB than in patients taking $\mathrm{G}+\mathrm{G} 300(P<0.0001)$. The betweengroup difference in highest post-breakfast glucose level $(\Delta=\mathrm{G}+\mathrm{G} 300-\mathrm{L}+\mathrm{GB})$ was correlated with $24-\mathrm{h}$ mean glucose level $(r=0.40, P=0.03)$.

\section{Conclusions}

It is of interest to note that compared with $\mathrm{L}+\mathrm{GB}, \mathrm{G}+$ G300 decreases postbreakfast glucose level and reduces nocturnal and 24-h glucose variability and level without causing hypoglycemia and daily variance.

\section{Comments}

To date, few studies have been published in which clinical aspects of BioIns are investigated. This study has an unusual study design, and the difference in the results observed might very well be explained by the small study size and short duration of the intervention. A clinically relevant difference between the originator basal insulin and the BioIns basal insulin cannot be concluded from this study (assuming that the two prandial insulins tested also have a very similar PD profile). Interestingly, this clinical study was conducted in a hospital in Japan but does not mention which BioIns of insulin glargine was studied.

\section{Efficacy and safety of biosimilar SAR342434 insulin lispro in adults with type 2 diabetes, also using insulin glargine: SORELLA 2 Study}

Derwahl $K M^{1}$, Bailey $T S^{2}$, Wernicke-Panten $K^{3}$, Ping $L^{4}$, Pierre $S^{5}$

${ }^{1}$ Institut fuer Klinische Forschung und Entwicklung (IKFE) Berlin GmbH, Berlin, Germany; ${ }^{2}$ AMCR Institute, Escondido, CA; ${ }^{3}$ Sanofi-Aventis Deutschland GmbH, Frankfurt, Germany; ${ }^{4}$ Sanofi, Bridgewater, NJ; ${ }^{5}$ Sanofi, Paris, France

Diabetes Technol Ther 2018; 20: 49-58

\section{Background}

The BioIns of the rapid-acting insulin analog insulin lispro (SAR-Lis) is compared with the originator insulin lispro (U100; Humalog; Ly-Lis) to compare efficacy, safety, and immunogenicity of both insulins in adult patients with type 2 diabetes mellitus (T2DM) treated with multiple daily injections using insulin glargine (GLA-100) as basal insulin.

\section{Methods}

This was a 6-month, randomized, open-label, phase 3 study in which insulin doses were adjusted to achieve fasting and 2-h postprandial glucose targets according to American Diabetes Association guidelines. Primary endpoint was the HbA1c change from baseline to week 26. Secondary endpoints included fasting plasma glucose (FPG), seven-point self-monitored plasma glucose profiles, hypoglycemic events, treatment-emergent adverse events (TEAEs), and anti-insulin antibodies (AIA).

\section{Results}

Five hundred and five patients were randomized (1:1) to multiple daily injections of SAR-Lis $(n=253)$ or Ly-Lis $(\mathrm{n}=252)$ plus once-daily GLA-100. Change in HbA1c, measured as least-square mean (standard error), from baseline to week 26 was similar with both insulin lispro versions: 
SAR-Lis, $-0.92 \%(0.051)$ and Ly-Lis, $-0.85 \%$ (0.051). Noninferiority at prespecified $0.3 \%$ noninferiority margin was demonstrated. Similar changes were recorded for both groups in FPG, seven-point self-monitored plasma glucose profiles, including postprandial glucose excursions and mean glucose over $24 \mathrm{~h}$, and insulin dosages were observed.

\section{Conclusions}

This study in patients with T2DM using multiple daily injections showed similar efficacy and safety (including immunogenicity) for both insulin lispro versions.

\section{Comment}

The results of this study in patients with T2DM are in line with previously published data of patients with T1DM (18). Study results showed no difference in a number of clinical outcome parameters between the BioIns and the originator insulin lispro.

\section{Safety of insulin lispro and a biosimilar insulin lispro when administered through an insulin pump}

Thrasher $\mathrm{J}^{1}$, Surks $\mathrm{H}^{2}$, Nowotny $\mathrm{I}^{3}$, Pierre $\mathrm{S}^{4}$, Rotthaeuser $B^{3}$, Wernicke-Panten $K^{3}$, Garg $S^{5}$

${ }^{I}$ Medical Investigations, Inc, Little Rock, AR; ${ }^{2}$ Sanofi, Bridgewater, NJ; ${ }^{3}$ Sanofi-Aventis Deutschland GmbH, Frankfurt, Germany; ${ }^{4}$ Sanofi, Paris, France; ${ }^{5}$ Barbara Davis Center for Diabetes, University of Colorado Denver, Aurora, $\mathrm{CO}$

\section{J Diabetes Sci Technol 2018; 12: 680-686}

\section{Background}

A BioIns (U100; SAR-Lis) of insulin lispro (U100; LyLis) was studied, assuming similar pharmacokinetics/pharmacodynamics between the two products (this has been demonstrated in a hyperinsulinemic euglycemic clamp study). Safety of both insulin lispros was studied when administered by continuous subcutaneous insulin infusion (CSII).

\section{Methods}

A randomized, open-label, $2 \times 4$-week, two-arm crossover study was carried out in 27 patients with T1D. Incidence of infusion set occlusions (ISOs) was the main outcome. Intervals between infusion set changes, TEAEs (including infusion site), hypersensitivity reactions and hypoglycemic events, and safety were secondary outcomes.

\section{Results}

A limited number of patients reported at least one ISO: 6/ 25 patients on SAR-Lis reported 14 ISOs and 4/27 on Ly-Lis reported 9 ISOs. The estimated difference in ISO risk between the two insulin lispro versions was $7.9 \%$ (95\% CI - 1.9 to 17.7). SAR-Lis and Ly-Lis had similar mean time between infusion set changes (3.1 and 3.0, respectively) as well as a similar event rate (events/patient-month) of any hypoglycemia (7.2 and 8.0, respectively). The percentage of patients who experienced any TEAE was also similar between the two versions $(12.0 \%$ vs $14.8 \%)$.

\section{Conclusion}

Both insulin lispro versions were well tolerated by patients using insulin pumps. The results do not imply a clinically significant difference in the risk of ISO for either insulin lispro when used with CSII.

\section{Comments}

This is the first clinical study in which a BioIns was compared with the originator insulin in patients with diabetes using an insulin pump. It is encouraging that both insulin lispro versions were well tolerated; however, this study included only a small number of participants and had short duration of treatment.

\section{Anti-insulin antibodies and adverse events with biosimilar insulin lispro compared with Humalog insulin lispro in people with diabetes}

Home $P^{1}$, Derwahl $\mathrm{KM}^{2}$, Ziemen $\mathrm{M}^{3}$, Wernicke-Panten $K^{3}$, Pierre $\mathrm{S}^{4}$, Kirchhein $Y^{3}$, Garg $\mathrm{SK}^{5}$

${ }^{1}$ Institute for Cellular Medicine, Newcastle University, Newcastle upon Tyne, UK; ${ }^{2}$ Institut fuer Klinische Forschung und Entwicklung, Berlin, Germany; ${ }^{3}$ Sanofi-Aventis Deutschland, Frankfurt, Germany; ${ }^{4}$ Sanofi, Paris, France; ${ }^{5}$ Barbara Davis Center for Diabetes, University of Colorado Denver, Aurora, $\mathrm{CO}$

Diabetes Technol Ther 2018; 20: 160-170

\section{Background}

The immunological properties of a BioIns of insulin lispro (SAR-Lis) of insulin lispro (Ly-Lis) were compared in two randomized, controlled, open-label, parallel-group, phase 3 studies, which were conducted to compare the efficacy and safety of both insulin lispro versions (both in combination with insulin glargine). The SORELLA 1 study was a 12- month study in 507 people with type 1 diabetes mellitus (T1DM), and the SORELLA 2 study was a 6-month study in 505 people with type 2 diabetes mellitus (T2DM).

\section{Methods}

Anti-insulin antibodies (AIA) were measured regularly throughout both studies at a centralized laboratory blinded to treatment groups using a drug-specific AIA assay. The AIA status (positive or negative), AIA titers, and cross-reactivity to human insulin, insulin glargine, and insulin glargine metabolite M1 were analyzed. The potential effect of AIA on safety, particularly as related to hypersensitivity reactions, hypoglycemia, and treatment-emergent adverse events, as well as on glycemic control (HbAlc, insulin dose), was also evaluated.

\section{Results}

AIA-positive status at baseline was similar for the two insulins, but higher in T1DM than in T2DM. In both studies, the percentage of people newly developing AIA in the two 
treatment groups or having a $\geq 4$-fold increase in AIA titers did not differ. No relationship was observed between maximum individual AIA titers and change in HbA1c or insulin dose, hypoglycemia, or hypersensitivity reactions or between efficacy/safety measures and subgroups by presence or absence of treatment-emergent AIA. Hypersensitivity events and events adjudicated as allergic reactions were few and did not differ between the two groups.

\section{Conclusion}

Both insulin lispro versions had a similar immunogenicity profile in patients with T1DM or T2DM.

\section{Comment}

It is of interest to note that in the large clinical trials performed with this BioIns, a detailed and careful analysis of the immunological properties showed no difference in comparison with the originator insulin.

\section{Cross-sectional survey of biosimilar insulin utilization in Asia: The Joint Asia Diabetes Evaluation Program}

Gani $L^{1,2}$, Lau $E^{2}$, Luk $A^{3}$, Sobrepena $L^{4}$, Tran $Q K^{5}$, Kesavadev $J^{6}$, Jia $W^{7}, Y u W^{8}$, Tsang $C^{9}$, Mukhopadhyay $M^{10}$, Jha $S^{11}$, Sheu $W^{12}$, Ho $Y K^{13}$, Nguyen $T K^{14}$, Ozaki $R^{3}$, So $W Y^{3}$, Kwan $C^{2}, F u{ }^{2} A C^{2}$, Mirasol $R^{15}$, Phatak $S R^{16}$, Kumar $K M P^{17}$, Aravind $S^{18}$, Janakiraman $H^{19}$, Chan JCN ${ }^{2,3}$; on behalf of the JADE Collaborative Study Group

${ }^{1}$ Changi General Hospital, Singapore; ${ }^{2}$ Asia Diabetes Foundation, Prince of Wales Hospital; ${ }^{3}$ Department of Medicine and Therapeutics, Hong Kong Institute of Diabetes and Obesity, Prince of Wales Hospital, The Chinese University of Hong Kong, Sha Tin, Hong Kong; ${ }^{4}$ Heart of Jesus Hospital, San Jose, Philippines; ${ }^{5}$ Nguyen Tri Phuong Hospital, Ho Chi Minh, Vietnam; 'Jothydev's Diabetes and Research Center, Thiruvananthapuram, Kerala, India; ${ }^{7}$ Shanghai Sixth People's Hospital, Shanghai; ${ }^{8}$ Huaian Second People's Hospital, Jiangsu, China; ${ }^{9}$ Alice Ho Nethersole Hospital, Tai Po, Hong Kong; ${ }^{10} \mathrm{Dr}$. M.K. Mukhopadhyay's Diabetic Clinic, Kolkata, West Bengal, India; ${ }^{11}$ Max Super Specialty Hospital, New Delhi, Delhi, India; ${ }^{12}$ Taichung Veterans General Hospital, Taichung, Taiwan; ${ }^{13}$ The Catholic University of Korea, Seoul, Korea; ${ }^{14}$ Medic Medical Center, Ho Chi Minh, Vietnam; ${ }^{15}$ Manila Doctors Hospital, Manila, Philippines; ${ }^{16}$ Vijayratna Diabetes Diagnosis and Treatment Centre, Ahmedabad, Gujarat, India; ${ }^{17}$ Center for Diabetes and Endocrine Care, Bengaluru, Karnataka, India; ${ }^{18}$ Diacon Hospital, Bengaluru, Karnataka, India; ${ }^{19}$ Salem Gopi Hospital, Salem, Tamil Nadu, India

J Diabetes Investig 2018; 9: 1312-1322

\section{Background}

The basic assumption with BioIns is that their usage will reduce treatment costs, although the extent of their use is largely unknown. By means of a register, BioIns use and its associations with the quality of glycemic control were evaluated in Asia.

\section{Materials and Methods}

A cross-sectional analysis was carried out in 81,531 patients with T1D and T2D enrolled into the register from 2007 to 2014. A multivariate analysis was performed to assess the association of BioIns compared with originator insulin with dosage, HbA1c, and hypoglycemia events.

\section{Results}

Among all patients, $20.5 \%(n=16,738)$ were treated with insulin. In four countries with high use of BioIns, $4.7 \%$ $(n=719)$ of insulin users $(n=10,197)$ were treated with BioIns (India, $\mathrm{n}=507,70.3 \%$; the Philippines, $\mathrm{n}=90,12.5 \%$; China, $n=62,8.6 \%$; Vietnam, $n=60,8.3 \%$ ). Compared with users of originator insulin, BioIns users were younger, with higher body mass index, HbA1c, and insulin dosage as well as a higher rate of hypoglycemia. None of these associations were significant after adjusting for confounders. The only factors independently associated with these measures were age, college education, diabetes education, lipid control, physical activity, and history of cardiovascular complications.

\section{Conclusions}

In Asia, the use of BioIns is not uncommon, and the data exclusion due to incomplete brand-name information suggests a possibly higher level of BioIns use. Because the quality of glycemic control is influenced by multiple determinants, prospective cohorts and diabetes registers should be established to monitor the safety and efficacy of different BioIns brands as well as their effects on clinical outcomes.

\section{Comments}

At first glance, this appears to be an interesting manuscript with real-world data from many patients; however, a close look reveals many issues that call into question the results presented. A number of questions arise, including selection bias, the relatively small number of BioIns users (mainly in India), the use of different BioIns in different countries, the cross-sectional nature of the evaluation, etc.

\section{Comparative evaluation of pharmacokinetics and pharmacodynamics of insulin glargine (Glaritus) and Lantus: a double-blind, randomized clamp study}

Bhatia $A^{1}$, Tawade $S^{1}$, Mastim $M^{1}$, Kitabi $E N^{2}$, Gopalakrishnan $M^{2}$, Shah $M^{1}$, Yeshamaina $S^{1}$, Gobburu $J^{2}$, Sahib $M^{3}$, Thakur $D^{3}$, Prasanna Kumar $K^{4}{ }^{4}$

${ }^{1}$ Wockhardt, Global Clinical Development, BKC, Mumbai, India; ${ }^{2}$ School of Pharmacy, Center for Translational Medicine, University of Maryland, Baltimore, MD; ${ }^{3}$ Wockhardt, Research Center, Aurangabad, India; ${ }^{4}$ Centre for Diabetes and Endocrine Care, Bengaluru, India

Acta Diabetol 2018 55: 461-468 


\section{Background}

The PK and PD properties of a BioIns of insulin glargine (Glaritus) and the originator formulation (Lantus; reference) were compared in a euglycemic glucose clamp study.

\section{Methods}

In this single-dose, double-blind, randomized, twosequence, four-period replicate crossover study in healthy volunteers $(n=40)$, subjects received subcutaneous administration of the two different insulin glargine versions (0.4 IU/ $\mathrm{kg}$ ) at two occasions. Glucose was infused at variable rate to maintain euglycemia for $24 \mathrm{~h}$.

\section{Results}

Endpoints for both PK (area under the curve insulin concentrate and maximum insulin concentration) and PD (area under the glucose infusion rate time curve and maximum glucose infusion rate) were comparable between both insulin glargine versions; the $90 \%$ confidence interval of geometric mean ratio of test to reference was entirely contained within $0.80-1.25$. Both formulations showed equivalent geometric least-square mean $(0.08 \mathrm{nmol} / \mathrm{L})$ for maximum insulin concentration. Also, the AUC0-24h values were comparable for both insulin glargines (1.09 vs $1.05 \mathrm{~h} \mathrm{nmol} / \mathrm{L})$. Median time to maximal insulin values were also identical ( $12 \mathrm{~h}$ for both), and median time to half-maximal values were also equal ( $18 \mathrm{~h}$ for both). No adverse events related to insulin glargine formulations were reported, both products were well tolerated.

\section{Conclusions}

Both insulin glargine versions showed similar PK and PD properties in this study.

\section{Comments}

The insulin glargine BioIns studied in this glucose clamp study was manufactured in India, and the clamp study was performed there as well. The sample size studied was considerably (and surprisingly) lower than in other glucose clamp studies performed in other centers with BioIns of long-acting insulin analogs. This study was performed in healthy subjects, in contrast to the recommendations by, for example, the European Medical Agency (EMA) to perform such studies in patients with T1D. The study was already performed in 2015 and 2016. No details of the glucose clamp study procedure were presented. In summary, the data of this study must be interpreted with care.

\section{SMART INSULINS: NEW CONCEPTS AND DELIVERY SYSTEMS}

\section{Engineering glucose responsiveness into insulin}

Kaarsholm NC, Lin S, Yan L, Kelly T, van Heek $M$, Mu J, Wu M, Dai G, Cui Y, Zhu Y, Carballo-Jane E, Reddy V, Zafian P, Huo P, Shi S, Antochshuk V, Ogawa A, Liu F, Souza SC, Seghezzi W, Duffy JL, Erion $M$, Nargund RP, Kelley DE
Merck Research Laboratories, Merck \& Co., Inc., Kenilworth, NJ

\section{Diabetes 2018; 67: 299-308}

\section{Abstract}

Insulin has a narrow therapeutic index, and because of this, the margin between a dose that achieves good glycemic control and one that causes hypoglycemia is quite small. Once injected, the clearance of exogenous insulin is invariant regardless of blood glucose, aggravating the potential to cause hypoglycemia. The aim of this work was to create a "smart" insulin that can modify insulin clearance, and therefore insulin action, in response to blood glucose level, decreasing the risk of hypoglycemia. Saccharide units were added to insulin, creating insulin analogs with affinity for both the insulin receptor (IR) and the mannose receptor C-type 1 (MR), which clears endogenous mannosylated proteins, a mechanism used to create glucose responsivity in insulin analogs. Iteration of this process resulted in the discovery of MK-2640, along with its in vitro and in vivo preclinical properties detailed in this report. In glucose clamp experiments using healthy dogs, as plasma glucose decreased stepwise from $280 \mathrm{mg} / \mathrm{dL}$ to $80 \mathrm{mg} / \mathrm{dL}$, progressively more MK-2640 was cleared via MR, reducing its availability for binding to the IR by $\sim 30 \%$. Dose escalation studies in diabetic minipigs found a higher therapeutic index for MK-2640 (3fold) vs regular insulin (1.3-fold).

\section{Synthetic beta cells for fusion-mediated dynamic insulin secretion}

Chen $Z^{1,2}$, Wang $J^{1,2}$, Sun $W^{1,2}$, Archibong $E^{1}$, Kahkoska $A R^{3}$, Zhang $X^{1,2}$, Lu $Y^{1,2}$, Ligler $\mathrm{FS}^{1}$, Buse $\mathrm{JB}^{3}$, Gu $Z^{1,2,3}$

${ }^{1}$ Joint Department of Biomedical Engineering, University of North Carolina at Chapel Hill and North Carolina State University, Raleigh, NC; ${ }^{2}$ Division of Pharmacoengineering and Molecular Pharmaceutics and Center for Nanotechnology in Drug Delivery, Eshelman School of Pharmacy, University of North Carolina at Chapel Hill, Chapel Hill, NC; ${ }^{3}$ Department of Medicine, University of North Carolina School of Medicine, Chapel Hill, NC

Nat Chem Biol 2018; 14: 86-93

\section{Abstract}

Artificial pancreatic beta cells that can robustly mimic glucose-responsive insulin secretion could provide a more user-friendly method for improving clinical outcomes in people with diabetes. This article details the use of synthetic materials to construct artificial beta cells $(\mathrm{A} \beta \mathrm{Cs})$ with a multicompartmental "vesicles-in-vesicle" superstructure that includes a glucose-metabolism system and membrane-fusion machinery. Using a sequential cascade of glucose uptake, enzymatic oxidation, and proton efflux, the A $\beta$ Cs can effectively discern high vs normal glucose levels. High glucose uptake and oxidation during hyperglycemic episodes decrease $\mathrm{pH}(<5.6)$, which in turn induces steric deshielding of peptides tethered to the insulin-loaded small, inner liposomal vesicles. These vesicle peptides then form coils with the complementary peptides attached to the inner surfaces of large vesicles, which brings the membranes of the inner and outer vesicles together, triggering their fusion and insulin "exocytosis." 


\section{Multiresponsive supramolecular theranostic nanoplatform based on pillar[5]arene and diphenylboronic acid derivatives for integrated glucose sensing and insulin delivery}

Zuo $M^{1}$, Qian $W^{1}, X u Z^{1}$, Shao $W^{l}, H u X^{1,2}$, Zhang $D^{3}$, Jiang $J^{1}$, Sun $X^{4}$, Wang $L^{1,4}$

${ }^{1}$ Key Laboratory of Mesoscopic Chemistry of MOE and Collaborative Innovation Center of Chemistry for Life Sciences, School of Chemistry and Chemical Engineering, Nanjing University, Nanjing, China; ${ }^{2}$ Applied Chemistry Department, School of Material Science \& Engineering, Nanjing University of Aeronautics \& Astronautics, Nanjing, China; ${ }^{3}$ State Key Laboratory of Pharmaceutical Biotechnology, School of Life Science, Nanjing University, Nanjing,China; ${ }^{4}$ School of Petrochemical Engineering, Changzhou University, Changzhou, China

Small 2018; 14 : e1801942

\section{Abstract}

A closed-loop "smart" insulin delivery system with the ability to mimic pancreatic cells will be highly desirable for diabetes treatment. This study details an insulin delivery platform based on an explicit supramolecular strategy that can respond to multiple stimuli. Insulin-glucose oxidase (GOx)-loaded supramolecular vesicles are self-assembled from a well-designed amphiphilic host-guest complex formed by pillar[5]arene and a derivative of diphenylboronic acid derivative, which is then loaded with insulin and GOx. These supramolecular vesicles can selectively recognize glucose, with structure disruption and efficient release of the entrapped insulin triggered by high glucose concentration as well as the in situ generated hydrogen peroxide and acidic microenvironment resulting from the GOx-promoted specific oxidation of glucose into gluconic acid. Moreover, this "smart" supramolecular theranostic nanoplatform is capable of functioning as both a glucose sensor and a controlled insulin delivery actuator. In addition, this smart supramolecular nanocarrier rapidly responded to hyperglycemic circumstances and effectively regulated the glucose levels during in vivo experiments using a mouse model of T1D.

\section{Core-shell microneedle gel for self-regulated insulin delivery}

Wang $J^{1,2}$, Ye $Y^{1,2}, Y u J^{1,2}$, Kahkoska $A R^{3}$, Zhang $X^{1,2}$, Wang $C^{1,2}$, Sun $W^{1,2}$, Corder $R D^{4}$, Chen $Z^{1,2}$, Khan $S^{4}{ }^{4}$ Buse $J B^{3}, G u Z^{1,2,3}$

${ }^{1}$ Joint Department of Biomedical Engineering, University of North Carolina at Chapel Hill and North Carolina State University, Raleigh, NC; ${ }^{2}$ Division of Molecular Pharmaceutics and Center for Nanotechnology in Drug Delivery, Eshelman School of Pharmacy, University of North Carolina at Chapel Hill, Chapel Hill, NC; ${ }^{3}$ Department of Medicine, University of North Carolina School of Medicine, Chapel Hill, NC; ${ }^{4}$ Department of Chemical and Biomolecular Engineering, North Carolina State University, Raleigh, $N C$

ACS Nano 2018; 12: 2466-2473

\section{Abstract}

Self-regulation of blood glucose using a bioinspired glucose-responsive insulin delivery system could improve health and quality of life outcomes for patients with T1D and advanced T2D. This paper describes a core-shell microneedle array patch that consists of degradable cross-linked gel offering smart insulin delivery with painless, rapid responsiveness and excellent biocompatibility. A covalently attached glucose-specific enzyme inside the gel oxidizes glucose, generating hydrogen peroxide $\left(\mathrm{H}_{2} \mathrm{O}_{2}\right)$ that triggers partial dissociation of the gel-based patch and subsequent release of insulin. Importantly, the $\mathrm{H}_{2} \mathrm{O}_{2}$-responsive microneedles have a thin coating embedded with $\mathrm{H}_{2} \mathrm{O}_{2}$-scavenging enzyme, mimicking the complementary function of peroxisome enzymes and protecting normal tissues from damage caused by oxidative stress. A chemically induced T1D mouse model was used to demonstrate that this smart insulin patch with a bioresponsive core and protective shell could offer improved biocompatibility and effectively regulate blood glucose levels within a normal range.

\section{Synthetic "smart gel" provides glucose-responsive insulin delivery in diabetic mice}

Matsumoto $A^{1}$, Tanaka $M^{2}$, Matsumoto $H^{1}$, Ochi $K^{2}$, Moro-Oka $Y^{1}$, Kuwata $H^{2,3}$, Yamada $H^{1}$, Shirakawa I ${ }^{4}$, Miyazawa $T^{1}$, Ishii $H^{3}$, Kataoka $K^{5,6}$, Ogawa $Y^{7,8,9}$, Miyahara $Y^{1}$, Suganami $T^{2}$

${ }^{1}$ Institute of Biomaterials and Bioengineering, Tokyo Medical and Dental University, Tokyo, Japan; ${ }^{2}$ Department of Molecular Medicine and Metabolism, Research Institute of Environmental Medicine, Nagoya University, Nagoya, Japan; ${ }^{3}$ Department of Diabetology, Nara Medical University, Nara, Japan; ${ }^{4}$ Department of Organ Network and Metabolism, Graduate School of Medical and Dental Sciences, Tokyo Medical and Dental University, Tokyo, Japan; ${ }^{5}$ Innovation Center of NanoMedicine, Kawasaki Institute of Industrial Promotion, Kawasaki, Japan; ${ }^{6}$ Policy Alternatives Research Institute, University of Tokyo, Tokyo, Japan; ${ }^{7}$ Department of Molecular Endocrinology and Metabolism, Graduate School of Medical and Dental Sciences, Tokyo Medical and Dental University, Tokyo, Japan; ${ }^{8}$ Department of Medicine and Bioregulatory Science, Graduate School of Medical Sciences, Kyushu University, Fukuoka, Japan; 9 Japan Agency for Medical Research and Development, Core Research for Evolutional Science and Technology, Tokyo, Japan

Sci Adv 2017; 3: eaaq0723

\section{Abstract}

Numerous previous attempts have been made to create protein-based, electronics-free insulin delivery systems employing glucose oxidase and sugar-binding lectins as a mechanism for glucose-sensing; however, these proteinbased materials are intolerant of long-term use and storage due to their denaturing and/or cytotoxic properties, rendering these clinical translations unsuccessful. This study created a solution by designing a protein-free, entirely synthetic material-based approach. The sugar-responsive properties of boronic acid were used to create a synthetic polymer gelbased insulin delivery device that is housed within a single 
catheter and demonstrates an artificial pancreas-like function in vivo. The device was subcutaneously implanted in healthy diabetic mice, where it established a closed-loop system comprising "continuous glucose sensing" and "skin layer"regulated insulin release. The system successfully controlled glucose metabolism in response to interstitial glucose fluctuation under both insulin-deficient and insulin-resistant conditions with 3 weeks or more of durability. This "smart gel" technology could provide a user-friendly and economical (disposable) alternative to the current state of the art systems, increasing the availability of effective insulin treatment to diabetic patients in developing countries as well as patients such as the elderly, infants, and those in need of nursing care, who otherwise might not be strongly motivated.

\section{Comments}

As we noted in the 2017 yearbook, glucose-responsive "smart" insulins continue to move toward clinical implementation. Kaarsholm et al. published an overview of their preclinical data, but although the phase 1 human trial from Merck with those compounds was completed in August 2016, up to now no human data have been published. The artificial cell approach described by Chen et al. is appealing because the reversible nature of $\mathrm{pH}$ responsive attachment and detachment of the inner vesicles can occur multiple times in response to blood glucose fluctuations. Nevertheless, the major limiting factor remains the need for constant replenishment of the insulin, which would necessitate periodic injections. Therefore, the same group works on the development of a smart insulin skin patch as described in the paper by Wang et al. Smart insulin delivery systems have also been suggested by Zuo et al., with a supramolecular theranostic nanoplatform or a smart insulin gel developed by Matsumoto et al. It must be noted that all of these systems so far have proven to be effective only in the mouse model, and it is likely that considerable work is needed before human studies are attempted (19).

\section{RAPID AND ULTRA-RAPID INSULINS: EVOLVING DATA}

\section{Efficacy and safety of rapid-acting insulin analogs in special populations with type 1 diabetes or gestational diabetes: systematic review and meta-analysis}

Norgaard $K^{1,2}$, Sukumar $N^{3}$, Rafnsson $S B^{4,5}$,
Saravanan $P^{3,6}$

${ }^{1}$ Department of Endocrinology, Copenhagen University Hospital Hvidovre, Hvidovre, Denmark; ${ }^{2}$ Steno Diabetes Center Copenhagen, Gentofte, Denmark; ${ }^{3}$ Diabetes, Endocrinology and Metabolism, Division of Health Sciences, Warwick Medical School, University of Warwick, Coventry, $U K ;{ }^{4}$ Centre for Primary Health and Social Care, London Metropolitan University, London, UK; ${ }^{5}$ Department of Epidemiology and Public Health, University College London, London, UK, ${ }^{6}$ Department of Diabetes, Endocrinology and Metabolism, George Eliot Hospital, Nuneaton, UK

Diabetes Ther 2018; 9: 891-917

\section{Introduction}

The efficacy and safety of three available rapid-acting insulin analogs (insulins lispro, aspart, and glulisine) were assessed in three groups of patients with T1D: pregnant women, children/adolescents, and people using CSII.

\section{Methods}

Electronic searches of the PubMed, EMBASE, and Cochrane Reviews databases were carried out to identify records for further study. Selected articles and their reference lists were examined to identify suitable studies for review and inclusion in a meta-analysis. Eligible studies were randomized controlled trials that reported data on relevant clinical outcomes. A unique reviewer was used for each of the three subpopulations. Each reviewer abstracted data their assigned subpopulation, and an additional reviewer abstracted data for all three. Any discrepancies between the reviewers were resolved by consensus or by consulting a fourth reviewer.

\section{Results}

People on CSII experienced greater reduction postprandial (postbreakfast) plasma glucose with rapid-acting insulin analogs than with regular human insulin (RHI) (mean difference $-1.63 \mathrm{mmol} / \mathrm{L}$ [95\% CI -1.71 to -1.54$]$ ), with similar risk of hypoglycemia and a trend for decreased HbA1c. Pediatric patients achieved comparable glycemic control and no safety concerns with both rapid-acting insulin analogs and RHI. Meta-analysis showed essentially no risk difference for severe hypoglycemic events between rapid-acting insulin analogs and RHI (risk difference 0.00 [95\% CI -0.01 to $0.01])$. In the pregnancy group, insulin lispro and insulin aspart were both found to be safe and effective for the mother as well as fetus, with glycemic control being at least as good as with RHI. No data were available for insulin glulisine during pregnancy.

\section{Conclusions}

Although additional trials would be beneficial, rapidacting insulin analogs appear to be generally safe and effective in these three special populations.

\section{A randomized comparison of three prandial insulin dosing algorithms for children and adolescents with type 1 diabetes}

Lopez $P E^{1,2,3}$, Evans $M^{4}$, King $B R^{1,2,3}$, Jones $T W^{4}$, Bell $K^{5}$, McElduff $P^{1,3}$, Davis $E A^{4}$, Smart $C E^{1,2,3}$

${ }^{1}$ Hunter Medical Research Institute, Newcastle, NSW, Australia; ${ }^{2}$ John Hunter Children's Hospital, Newcastle, NSW, Australia; ${ }^{3}$ University of Newcastle, Newcastle, NSW, Australia; ${ }^{4}$ Telethon Kids Institute, University of Western Australia, Perth, WA, Australia; ${ }^{5}$ University of Sydney, NSW, Australia

\section{Diabet Med 2018; 35: 1440-1447}

\section{Aim}

Two novel insulin-dosing algorithms (the Pankowska Equation and the Food Insulin Index) were systematically compared with carbohydrate counting to assess the relative 
effect of each on postprandial glucose excursions following both high-fat and high-protein meals.

\section{Methods}

A randomized, crossover trial was conducted in 33 children at two pediatric diabetes centers in the UK. Test meals contained a similar amount of carbohydrates, with a high amount of either fat or protein. On each day of the study, subjects consumed either a high-protein or high-fat meal. Insulin dosing was based on carbohydrate counting, the Pankowska Equation, or the Food Insulin Index. Participants fasted for $5 \mathrm{~h}$ after the test meal, and physical activity was standardized. Postprandial glycemia was measured for 300 min via continuous glucose monitoring.

\section{Results}

Compared with carbohydrate counting, the Pankowska Equation resulted in lower glycemic excursion for 90-240 min after the high-protein meal $(P<0.05)$ and lower peak glycemic excursion $(P<0.05)$. Carbohydrate counting and the Food Insulin Index significantly lowered the risk of hypoglycemia compared with the Pankowska Equation (odds ratios: 0.76 for carbohydrate counting vs Pankowska Equation and 0.81 for Food Insulin Index vs Pankowska Equation). There was no significant difference in glycemic excursions for carbohydrate counting vs the Food Insulin Index.

\section{Conclusion}

The Pankowska Equation resulted in reduced postprandial hyperglycemia yet also brought an increase in hypoglycemia. There were no significant differences between carbohydrate counting and the Food Insulin Index. Further research is required to optimize prandial insulin dosing.

\section{Efficacy and safety of fast-acting insulin aspart in comparison with insulin aspart in type 1 diabetes (onset 1): A 52-week, randomized, treat-to-target, phase III trial}

Mathieu $C^{1}$, Bode $B W^{2}$, Franek $E^{3}$, Philis-Tsimikas $A^{4}$, Rose $L^{5}$, Graungaard $T^{6}$, Birk $\emptyset$ sterskov $A^{7}$, Russell-Jones $D^{8}$

${ }^{1}$ Clinical and Experimental Endocrinology, University Hospital Leuven, Leuven, Belgium, ${ }^{2}$ Atlanta Diabetes Associates, Atlanta, GA; ${ }^{3}$ Mossakowski Clinical Research Centre, Polish Academy of Sciences, Warsaw, Poland; ${ }^{4}$ Scripps Whittier Diabetes Institute, Scripps Health, San Diego, CA; ${ }^{5}$ Institute of Diabetes Research, Münster, Germany; ${ }^{6}$ Biostatistics Aalborg 2, Novo Nordisk A/S, Aalborg, Denmark; ${ }^{7}$ Medical and Science, Insulin and Digital Health, Novo Nordisk A/S, Sфborg, Denmark; ${ }^{8}$ Diabetes and Endocrinology, Royal Surrey County Hospital and University of Surrey, Guildford, UK

Diabetes Obes Metab 2018; 20: 1148-1155

\section{Aims}

The safety and efficacy of fast-acting insulin aspart (faster aspart) was compared with conventional insulin aspart (IAsp) in adults with T1D.

\section{Methods}

This phase 3 , onset 1 trial was a 26 -week (initial treatment) plus 26-week (additional treatment), multicenter, randomized, treat-to-target trial was carried out at 165 sites across 9 countries. Adults with T1D were randomized to double-blind mealtime faster aspart or IAsp, both administered with onceor twice-daily insulin detemir. The primary endpoint was change in HbA1c from baseline at 26 weeks, has been reported previously. This publication reports results from the full 52-week study period.

\section{Results}

Between August 2013 and June 2015, participants were randomly assigned to double-blind faster aspart $(\mathrm{n}=381)$ or IAsp $(n=380)$. At the end of the two 26-week arms (52 weeks), estimated mean changes from baseline in $\mathrm{HbA} 1 \mathrm{c}$ levels were $-0.08 \%$ (faster aspart) and $+0.01 \%$ (IAsp); the estimated treatment difference significantly favored faster aspart $(-0.10 \%$ [95\% CI $-0.19 \%$ to $-0.00 \%$ ]; $P=0.0424)$. Faster aspart produced greater reductions from baseline in 1$\mathrm{h}$ postprandial plasma glucose (PPG) (meal test; faster aspart $-1.05 \mathrm{mmol} / \mathrm{L}$; IAsp $-0.14 \mathrm{mmol} / \mathrm{L}$ ) and was again favored by the estimated treatment difference $(-0.91 \mathrm{mmol} / \mathrm{L}[95 \%$ $\mathrm{CI}-1.40$ to -0.43$]$ vs $-16.48 \mathrm{mg} / \mathrm{dL}[95 \% \mathrm{CI}-25.17$ to 7.80]; $P=0.0002)$. There was no difference between treatments in treatment-emergent adverse events or in overall severe or blood glucose-confirmed hypoglycemic episodes.

\section{Conclusion}

In this study of patients with T1D, overall glycemic control at 52 weeks improved significantly with faster aspart vs IAsp, which is consistent with the findings at 26 weeks. With faster aspart, participants achieved an insulin profile closer to that of physiological insulin secretion, meaning lower PPG and HbA1c levels compared with those achieved with Iasp.

\section{Investigation of pump compatibility of fast-acting insulin aspart in subjects with type 1 diabetes}

Zijlstra $E^{l}$, Demissie $M^{2}$, Graungaard $T^{2}$, Heise $T^{l}$, Nosek $L^{1}$, Bode $B^{3}$

${ }^{1}$ Profil, Neuss, Germany; ${ }^{2}$ Novo Nordisk A/S, Sфborg, Denmark; ${ }^{3}$ Atlanta Diabetes Associates, Atlanta, GA

J Diabetes Sci Technol. 2018; 12: 145-151

\section{Background}

Ultra-fast-acting insulins, such as fast-acting insulin aspart (faster aspart), exhibit pharmacokinetic properties that may be beneficial for patients with T12D using CSII, if they are compatible with and safe to use in CSII. This onset 4 trial investigated the compatibility, safety, and short-term effectiveness, of faster aspart and insulin aspart in CSII therapy in adults with T1D.

\section{Methods}

This randomized, double-blind, parallel-group, actively controlled trial evaluated the compatibility, efficacy, and safety of faster aspart in adult patients with T1D at two centers in Germany and the United States. In both groups, 
participants used their own MiniMed Paradigm pump with Quick-Set or Silhouette infusion sets. Following a 2-week runin, subjects were randomly assigned (2:1) to faster aspart $(n=25)$ or insulin aspart $(n=12)$ for the 6-week treatment period. The primary endpoint was defined as the number of microscopically confirmed episodes of infusion-set occlusions.

\section{Results}

There were no microscopically confirmed episodes of infusion-set occlusions in either arm. Five participants (all using faster aspart) reported a total of seven possible infusion-set occlusions. None of the reports were attributed to a participant-observed plug (six were prompted by unexplained hyperglycemia; and one by leakage), and none were confirmed. Neither macroscopic nor microscopic evaluation revealed any color change or particle/crystal formation in the infusion sets. Premature infusion-set changes were reported in $44 \%$ of participants in the faster aspart group and $16.7 \%$ of the aspart group. The faster aspart group showed a nonsignificant trend toward better efficacy (estimated treatment difference HbA1c change: $-0.14 \%$ [95\% CI $-0.40 \%$ to $0.11 \%]$ ). No new safety issues were discovered in either group.

\section{Conclusions}

No microscopically confirmed infusion-set occlusions were observed for either faster aspart or insulin aspart during the 6-week treatment period, indicating that the two insulins have similar compatibility with CSII use.

\section{The value of fast-acting insulin aspart compared with insulin aspart for patients with diabetes mellitus treated with bolus insulin from a UK health care system perspective}

Leelarathna $L^{1}$, Ashley $D^{2}$, Fidler $C^{3}$, Parekh $W^{4}$

${ }^{I}$ Manchester Diabetes Centre, Central Manchester University Hospitals NHS Foundation Trust, Manchester, UK; ${ }^{2}$ Novo Nordisk Ltd, Gatwick, UK; ${ }^{3} D R G$ Abacus, Bicester, UK; ${ }^{4}$ Novo Nordisk Ltd, Gatwick, West Sussex, Surrey, UK

Ther Adv Endocrinol Metab 2018; 9: 187-197

\section{Background}

Fast-acting insulin aspart is a new formulation of the rapidacting insulin analogue insulin aspart, representing an advancement in onset of action and postprandial glucose control compared with current rapid-acting insulin analogues. The objective of the current study was to analyze the cost and highlight the value of prescribing fast-acting insulin aspart rather than insulin aspart, for patients with diabetes who require mealtime insulin.

\section{Methods}

A simple cost-impact analysis was carried out from the perspective of the UK National Health Service (NHS). The analysis included the cost of insulin but excluded out-ofpocket expenses incurred by patients as well as carers' costs and lost productivity. The time horizon of the analysis was 1 year; therefore, no discounting was applied.

\section{Results}

Switching from insulin aspart to fast-acting insulin aspart is cost neutral for the UK NHS. The cost of fast-acting insulin aspart is equivalent to that of insulin aspart for both vials and Penfill cartridges; fast-acting insulin aspart is also available in the FlexTouch pen at the same price as the insulin aspart FlexPen (making it effectively less expensive than the insulin aspart FlexTouch pen). Upon switching to fast-acting insulin aspart, patients using the insulin aspart FlexPen ${ }^{\circledR}$ will be upgraded to the FlexTouch ${ }^{\circledR}$ pen device, the preferred choice of both patients and health-care professionals, at no additional cost.

\section{Conclusions}

Fast-acting insulin aspart offers additional clinical benefit without additional cost compared with insulin aspart and therefore provides value to the UK NHS.

\section{Modeling subcutaneous absorption of fast-acting insulin in type 1 diabetes}

Schiavon M, Dalla Man C, Cobelli C

Department of Information Engineering, University of $\mathrm{Pa}$ dova, Padova, Italy

IEEE Trans Biomed Eng 2018; 65: 2079-2086

\section{Objective}

Subcutaneous (SC) administration of fast-acting insulin analogues is the key in conventional treatment of T1D. A SC insulin absorption model would be advantageous for optimizing insulin therapy and testing new open- and closed-loop treatment strategies in in silico platforms. To date, none of the published models have been assessed on a large, frequently sampled dataset of T1D subjects. The aim here is to propose a model of SC absorption of fast-acting insulin that is capable of describing the data and to precisely estimate model parameters with a clear physiological interpretation.

\section{Methods}

Three models were identified as candidates on 116 T1D subjects who underwent a single SC injection of fast-acting insulin. The models were compared based on their ability to describe the data and their numerical identifiability.

\section{Results}

The proposed linear, two-compartment model includes a subject-specific delay in SC insulin absorption. An average delay of $7.6 \mathrm{~min}$ in insulin appearance is detected in the first compartment. The insulin is then slowly absorbed into plasma (in $23 \%$ of the subjects) at a rate of $0.0034 \mathrm{~min}^{-1}$, while the remaining insulin diffuses into the second compartment, with a rate constant of $0.028 \mathrm{~min}^{-1}$. Finally, from the second compartment, the insulin is absorbed into plasma with a rate constant of $0.014 \mathrm{~min}^{-1}$.

\section{Conclusion}

Three models were tested, and only the one proposed here is capable of both accurately describing plasma insulin data 
after a single SC injection and precisely estimating physiologically plausible parameters. Further testing of the model is necessary, for cases of variable SC insulin delivery and/or multiple insulin doses.

\section{Significance}

The authors anticipate that these results will assist in the development of new open- and closed-loop insulin treatment strategies.

\section{Ultra-rapid BioChaperone lispro improves postprandial blood glucose excursions vs insulin lispro in a 14-day crossover treatment study in people with type 1 diabetes}

Andersen $G^{1}$, Meiffren $G^{2}$, Lamers $D^{1}$, DeVries $J H^{1}$, Ranson $A^{2}$, Seroussi $C^{2}$, Alluis $B^{2}$, Gaudier $M^{2}$, Soula $\mathrm{O}^{2}$, Heise $T^{l}$

${ }^{1}$ Profil, Neuss, Germany; ${ }^{2}$ Adocia, Lyon, France

Diabetes Obes Metab 2018; 20: 2627-2632

\section{Aims}

The safety and efficacy of BioChaperone Lispro (BCLIS), an ultra-rapid formulation of insulin lispro (LIS), was investigated in patients with T1D.

\section{Material and Methods}

This was a randomized, double-blind, crossover study consisting of two 14-day periods during which participants self-administered individualized bolus doses of BCLIS or LISventura. Postprandial blood glucose (BG) was tested after individualized solid mixed meal tests (MMTs) (50\% carbohydrate, $29 \%$ fat, $21 \%$ protein), with additional randomization for the timing of insulin administration, immediately $(\mathrm{t} 0), 15$ minutes prior to $(\mathrm{t}-15)$, and 15 minutes after $(\mathrm{t}+15)$ commencement of the meal on days 1,2 , and 3 , and with t0 administration on day 14. Pharmacokinetic (PK) variables were analyzed for t0 MMTs. Participants also administered individualized BCLIS or LIS doses immediately prior to meals during two 10-day outpatient periods with an unchanged basal insulin regimen.

\section{Results}

Thirty-five participants completed both treatment periods. In MMTs with t0 insulin administration, the higher early postprandial PK exposure of BCLIS was associated with significant reductions in 1- to 2 -h postprandial BG excursions by $30 \%-40 \%$ vs LIS, and the more rapid absorption and action of BCLIS persisted over 2 weeks. Glucose excursion over the full 360-minute postprandial period was unchanged. Postprandial BG control was similar for BCLIS injected at $t+15$ and LIS injected at $t 0$. The safety and tolerability of BCLIS was found to be similar to that of LIS. No injection site reactions occurred with BCLIS.

\section{Conclusions}

BCLIS was safe and well tolerated over the 14-day treatment period and significantly improved postprandial BG compared with LIS when administered at mealtime.

\section{Greater early postprandial suppression of endog- enous glucose production and higher initial glu- cose disappearance is achieved with fast-acting insulin aspart compared with insulin aspart}

Basu $A^{1}$, Pieber $T R^{2}$, Hansen $A K^{3}$, Sach-Friedl $S^{2}$, Erichsen $L^{3}$, Basu $R^{1}$, Haahr $H^{3}$

${ }^{1}$ Division of Endocrinology, University of Virginia, Charlottesville, VA; ${ }^{2}$ Division of Endocrinology and Diabetology, Department of Internal Medicine, Medical University of Graz, Graz, Austria; ${ }^{3}$ Novo Nordisk, Søborg, Denmark

Diabetes Obes Metab 2018; 20: 1615-1622

\section{Aims}

For patients with T1D, postprandial glucose (PPG) levels play an important role in attaining overall glycemic control, and fast-acting insulin aspart (faster aspart) has been formulated to provide better control of PPG. This study examined the mechanisms behind the lower PPG concentrations achieved with faster aspart compared with IAsp.

\section{Materials and Methods}

A randomized, single-center, double-blind, two-period crossover trial consisting of a screening visit, two dosing visits, and a follow-up visit was carried out in 41 adults with insulintreated T1D for $\geq 12$ months. At dosing visits, participants received identical subcutaneous single faster aspart and IAsp doses (individualized for each participant) along with a standardized mixed meal that included $75 \mathrm{~g}$ of carbohydrate labeled with $\left[1-{ }^{13} \mathrm{C}\right]$ glucose. PPG turnover was determined by the triple-tracer meal method using continuous, variable $\left[6-{ }^{3} \mathrm{H}\right]$ glucose and $\left[6,6-{ }^{2} \mathrm{H}_{2}\right]$ glucose intravenous infusion.

\section{Results}

During the first hour, insulin exposure was 32\% greater with faster aspart than with IAsp (treatment ratio, faster aspart/IAsp, 1.32 [95\% CI 1.18 to 1.48 ]; $P<0.001$ ), leading to a $0.59-\mathrm{mmol} /$ $\mathrm{L}$ nonsignificantly smaller PPG increment at 1 hour $\left(\Delta \mathrm{PG}_{1 \mathrm{~h}}\right.$; treatment difference, faster aspart - IAsp, $-0.59 \mathrm{mmol} / \mathrm{L}[95 \%$ CI -1.19 to 0.01$]$; $P=0.055)$. The trend toward decreased $\Delta \mathrm{PG}_{1 \mathrm{~h}}$ with faster aspart could be attributed to $12 \%$ greater suppression of endogenous glucose production (EGP; treatment ratio 1.12 [95\% CI 1.01 to 1.25$] ; P=0.040$ ) in addition to $23 \%$ higher first-hour glucose disappearance (1.23 [95\% CI 1.05 to 1.45]; $P=0.012$ ) with faster aspart than with IAsp. First-hour suppression of free fatty acid levels was $36 \%$ higher for faster aspart than for IAsp (1.36 [95\% CI 1.01 to 1.88 ]; $P=0.042)$.

\section{Conclusions}

The trend toward improved PPG control with faster aspart compared with IAsp in this study was attributable to both greater early suppression of EGP and stimulation of glucose disappearance.

\section{Comments}

Despite the introduction of ultra-rapid insulin into some countries over the last year, the vast majority of patients still use conventional rapid-acting analogs for their 
meal-time insulin coverage. Norgaard et al. presented a systematic review of the three available rapid-acting insulin analogs (insulins lispro, aspart, and glulisine) in special populations with T1D and concluded that they are safe and efficacious but there is a need for further studies. For example, Lopez et al. looked at the clinically very relevant question of whether carbohydrate counting alone is sufficient or whether the fat and protein content needs to be considered in meals with high protein or fat contents when calculating the prandial dose.

Ultra-rapid-acting insulins are intended to even better match the time-action profile of prandial insulins to cover the rapid increase in blood glucose after meals. In addition to the regulatory onset trials discussed in last year's article, the paper by Mathieu et al. provides evidence that the improved overall glycemic control is sustained at 52 weeks with lower PPG and HbA1c levels compared with those achieved with IAsp in people with T1D. Basu et al. used the triple-tracer meal method to demonstrate that the trend toward improved PPG control with faster aspart can be attributed to both greater early suppression of EGP and stimulation of glucose disappearance. As the ultra-rapidacting insulin may be particularly useful for pumps and "closed-loop" approaches, the 6-week compatibility data of Zijlstra et al. regarding FiAsp in pumps are reassuring, although the short duration and low patient numbers limit the value of this study. As "in-silico modelling" has become an FDA-approved way to test algorithms for automated-insulin delivery, the model of subcutaneous absorption of fast-acting insulin described by Schiavon et al. will help to speed up the implementation of ultrafast, rapid-acting insulin in the ongoing research. Pharmacoeconomic data presented by Leelarathna et al. indicated that a displacement of insulin aspart with FiAsp may be beneficial under the conditions of the UK NHS system, but clearly more real-world clinical data and similar costimpact analyses must be conducted from the perspective of other health delivery systems before more generalizable conclusions can be taken. Regarding the potential competitors for FiAsp, Andersen et al. published the first paper on Biochaperone lispro, but the future of this compound remains unclear, as discussed in the introduction. Finally, the year had another lesson on the need for extended patient handling testing and pharmacovigilance when a color change became necessary for the FiAsp label in order to avoid mix-ups with the light green label of the insulin degludec injection pen. The move followed reports of diabetes patients mistakenly injecting faster aspart instead of degludec, or the other way around, given that the light green and yellow may be difficult to distinguish from each other in low lighting (20).

\section{Author Disclosure Statement}

T.D. received speaker honoraria and research support and has consulted for Abbott, Bayer, BMS, AstraZeneca, Boehringer Ingelheim, Dexcom, Eli Lilly, Medtronic, Novo Nordisk, Sanofi, and Roche. He is a shareholder of DreaMed Ltd.

L.H. is a consultant for a number of insulin manufacturing companies.
J.B. has received honoraria for consulting and/or lecture fees from Abbott Diabetes Care, AstraZeneca, Eli Lilly, Novo Nordisk, and Sanofi.

\section{References}

1. Marso SM, McGuire DK, Zinman B, et al. Efficacy and safety of degludec versus glargine in type 2 diabetes. $N$ Engl J Med 2017; 377: 723-732.

2. Heise T, Nørskov M, Nosek L, et al. Insulin degludec: lower day-to-day and within-day variability in pharmacodynamics response compared with insulin glargine $300 \mathrm{U} /$ $\mathrm{mL}$ in type 1 diabetes. Diabetes Obes Metab 2017; 19: 1032-1039.

3. Monnier L, Collette C. Pharmacological variability of insulin degludec and glargine 300U/mL: equivalent or not? Diabetes Metab 2018; 44: 15-21

4. Becker RHA. Letter to the editor in response to Heise T, Nørskov M, Nosek L, et al. Insulin degludec: lower day-today and within-day variability in pharmacodynamics response compared with insulin glargine $300 \mathrm{U} / \mathrm{mL}$ in type 1 diabetes (Diabetes Obes Metab 2017; 19: 1032-1039). Diabetes Obes Metab 2018; 20: 2043-2047.

5. Heise T, Kaplan K, Haahr HL. Day-to-day and within-day variability in glucose-lowering effect between insulin degludec and insulin glargine $(100 \mathrm{U} / \mathrm{mL}$ and $300 \mathrm{U} / \mathrm{mL})$ : a comparison across studies. Diabetes Sci Technol 2018; 12: 356-363.

6. Heise T, Heckermann S, DeVries JH. Variability of insulin degludec and glargine $300 \mathrm{U} / \mathrm{mL}$ : a matter of methodology or just marketing? Diabetes Obes Metab 2018; 20: 20512056.

7. Cheng AYY, Rosenstock J, Ritzel R, et al. Similar glycemic control and less comparable hypoglycemia with insulin glargine $300 \mathrm{U} / \mathrm{mL}$ (Gla-300) vs degludec $100 \mathrm{U} / \mathrm{mL}$ (IDeg-100) in insulin-naïve T2DM on antihyperglycemic drugs \pm GLP-1 RAs - the BRIGHT randomized study. Diabetes 2018; 67 (Suppl 1): 301-OR.

8. Zhang X, Lam ECQ, Seger ME, et al. LY2963016 insulin glargine and insulin glargine (lantus) produce comparable pharmacokinetics and pharmacodynamics at two dose levels. Clin Pharmacol Drug Dev 2017; 6: 556-56

9. Crutchlow MF, Palcza JS, Mostoller KM, et al. Single-dose euglycaemic clamp studies demonstrating pharmacokinetic and pharmacodynamic similarity between MK-1293 insulin glargine and originator insulin glargine (Lantus) in subjects with type 1 diabetes and healthy subjects. Diabetes Obes Metab 2018; 20: 400-408.

10. Owens DR, Landgraf W, Schmidt A, et al. The emergence of biosimilar insulin preparations-a cause for concern? Diabetes Technol Ther 2012; 14: 989-996.

11. Carter AW, Heinemann L, Klonoff DC. Quality control of insulins and biosimilar insulins: what do we know? $J$ Diabetes Sci Technol 2016; 10: 811-815.

12. Carter AW, Heinemann L. Insulin concentration in vials randomly purchased in pharmacies in the United States: considerable loss in the cold supply chain. J Diabetes Sci Technol 2018; 12: 839-841.

13. Dowlat HA, Kuhlmann MK, Khatami H, et al. Interchangeability among reference insulin analogues and their biosimilars: regulatory framework, study design and clinical implications. Diabetes Obes Metab 2016; 18: $737-$ 746.

14. Heinemann L, Fritz I, Khatami H, Edelman SV. Administration of biosimilar insulin analogs: role of devices. Diabetes Technol Ther 2017; 19: 79-84. 
15. Davies M, Dahl D, Heise T, et al. Introduction of biosimilar insulins in Europe. Diabet Med 2017; 34: 1340-1353.

16. Tieu C, Lucas EJ, DePaola M, et al. Efficacy and safety of biosimilar insulins compared to their reference products: A systematic review. PLoS One 2018; 13: e0195012.

17. Yamada T, Kamata R, Ishinohachi K, et al. Biosimilar vs originator insulins: Systematic review and meta-analysis. Diabetes. Obes Metab 2018; 20: 1787-1792.
18. Garg SK, Wernicke-Panten K, Rojeski M et al. Efficacy and safety of biosimilar SAR342434 insulin lispro in adults with type 1 diabetes also using insulin glargine-SORELLA 1 study. Diabetes Technol Ther 2017;19: 516-526.

19. Hampton T. Smart artificial beta cells may help treat diabetes. JAMA 2018; 319: 11-12.

20. Safety alert: change of colour for fast-acting insulin Fiasp products. Drug Ther Bull 2018; 56: 62-63. 